\title{
Far-Field Plume Measurements of a Nested-Channel Hall-Effect Thruster
}

\author{
Raymond Liang* and Alec D. Gallimore ${ }^{\dagger}$ \\ University of Michigan, Ann Arbor, MI, 48109, USA
}

\begin{abstract}
Far-field plume measurements were performed on the X2 nested-channel Hall-effect thruster using a nude Faraday probe, retarding potential analyzer, and $\mathrm{E} \times \mathbf{B}$ probe. Data from these probes were used to examine the operating characteristics of the X2 with both channels operating simultaneously (dual-channel mode). For each operating condition in dual-channel mode, far-field measurements were also taken with each channel operating individually (single-channel mode) under the same applied magnetic field and cathode flow fraction. The results of the far-field measurements showed increased voltage utilization efficiency in dual-channel mode. Current utilization efficiency, divergence efficiency, charge utilization efficiency, and mass utilization efficiency did not show significant changes between the two operating modes. The far-field diagnostics also showed that the X2 operates similarly to a conventional Hall thruster when both channels are at the same discharge voltage. Small improvements in performance in the dual-channel mode were likely due to improved ion acceleration and reduced cathode coupling voltage.
\end{abstract}

\section{Nomenclature}

$$
\begin{array}{llll}
A_{c} & =\text { collector area } & \alpha_{L} & =\text { incidence angle relative to left centerline } \\
I_{\text {axial }} & =\text { axial beam current } & \alpha_{R} & =\text { incidence angle relative to right centerline } \\
I_{b} & =\text { beam current } & \beta & =\text { plume divergence half-angle } \\
I_{d} & \text { = discharge current } & \eta_{b} & =\text { current utilization } \\
\dot{m}_{a} & =\text { anode mass flow rate } & \eta_{c} & =\text { cathode efficiency } \\
\dot{m}_{c} & =\text { cathode mass flow rate } & \eta_{d} & =\text { divergence utilization efficiency } \\
\dot{m}_{t} & =\text { total mass flow rate } & \eta_{M} & =\text { total mass utilization efficiency } \\
N & =\text { number of nested channels } & \eta_{m} & =\text { mass utilization efficiency } \\
P_{d} & =\text { discharge power } & \eta_{m a g} & =\text { electromagnet efficiency } \\
P_{t} & =\text { total power } & \eta_{q} & =\text { charge utilization efficiency } \\
Q & =\text { average charge of ions } & \eta_{t} & =\text { total efficiency } \\
R & =\text { radial position } & \eta_{v} & =\text { voltage utilization efficiency } \\
R_{C L} & =\text { channel centerline radius } & \theta & =\text { angular position from thruster centerline } \\
R_{L} & =\text { probe distance from left centerline } & \kappa_{A} & =\text { area correction factor } \\
R_{R} & =\text { probe distance from right centerline } & \kappa_{D} & =\text { distance correction factor } \\
T & =\text { thrust } & \kappa_{G} & =\text { gap correction factor } \\
V_{a} & =\text { acceleration voltage } & \Omega & =\text { ion species current fraction } \\
V_{c} & =\text { cathode coupling voltage } & & \\
V_{d} & =\text { discharge voltage } & & \\
V_{m p} \quad & \text { most-probable voltage } & & \\
V_{p} & =\text { plasma potential } & & \\
Z & =\text { charge state } & &
\end{array}
$$

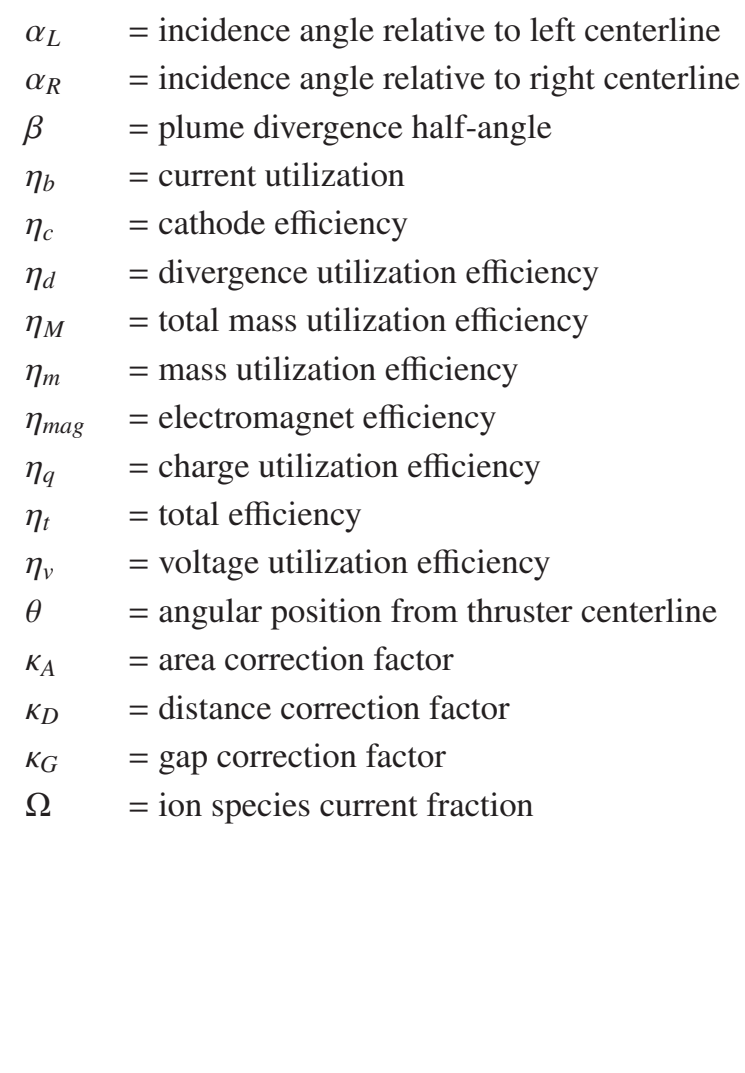

*Ph.D. Candidate, Aerospace Engineering, rayliang@umich.edu, and Student Member, AIAA.

${ }^{\dagger}$ Arthur F. Thurnau Professor, Aerospace Engineering, alec.gallimore@umich.edu, and Fellow, AIAA.

DISTRIBUTION STATEMENT A. Approved for public release; distribution is unlimited. 


\section{Introduction}

$\mathrm{T}$

HE Plasmadynamics and Electric Propulsion Laboratory (PEPL) at the University of Michigan and the Air Force

Research Laboratory have developed and tested a laboratory-model nested-channel Hall-effect thruster (NHT). Named the X2, the NHT was developed to determine the capabilities of the nested-channel configuration. The X2 features two concentric discharge channels with a shared internal cathode, as shown in Figure 1. Nesting is a relatively unexplored concept of clustering discharge channels of a Hall thruster. Available literature predating the X2 shows mostly conceptual consideration for NHTs with only a single mention of a development effort. ${ }^{1-3}$ Prior to this effort, the performance of an NHT had never been published, and the potential advantages have never been evaluated. Additionally, the properties of the plume of an NHT have never been determined and compared to those of traditional Hall thrusters.

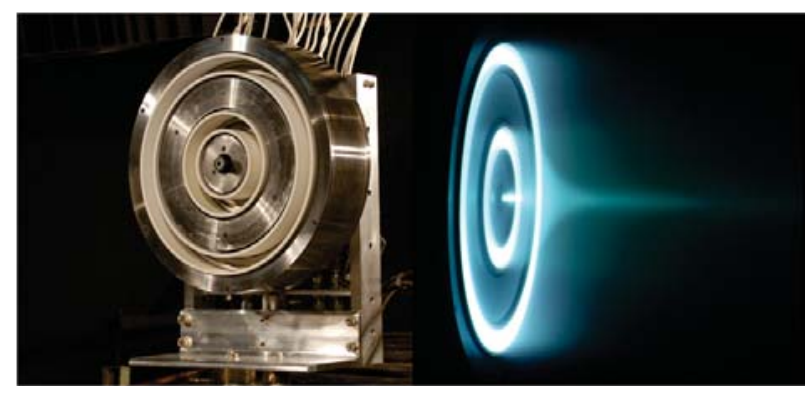

Figure 1: X2 nested-channel Hall-effect thruster

The nested-channel configuration has a number of advantages that make it particularly suitable for high-power electric propulsion. Studies have shown that nesting channels can lead to significant reductions in thruster mass and footprint area. ${ }^{1,4}$ Additionally, NHTs have the ability to change the exit area through the selective activation of available channels, which provides an additional method of throttling and a means of efficiently adapting to various levels of available spacecraft power. Multiple discharge channels also give a degree of system redundancy and permit multiple design points for the overall thruster.

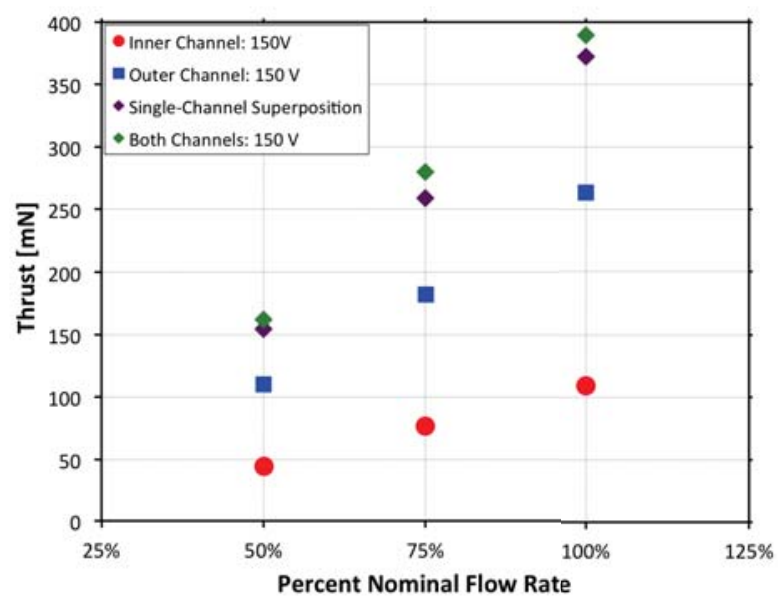

Figure 2: Comparison of thrusts from single-channel and dual-channel modes. The operating points with the same nominal flow rate percentages were tested at the same background pressure to reduce facility effects. The thrust uncertainty was approximately $\pm 2 \mathbf{~ m N}$.

Operation of the X2 with each channel firing individually (single-channel mode) and two channels firing simultaneously (dual-channel mode) has already been demonstrated. Slight improvements in performance in dual-channel mode were observed in previous measurements, although the cause of the increased thrust was unknown. Elevated background pressure was eliminated as a significant source of performance gains after single-channel operating conditions were conducted at the same background pressure as the corresponding dual-channel condition. As shown in Figure 2, the dual-channel thrusts were still approximately 5\% greater than the superposition single-channel thrusts 
when the applied magnetic fields, cathode flow fractions, and background pressures were held constant. Additional measurements to further investigate the dual-channel mode of the X2 were natural and necessary.

The purpose of this study is to investigate how the operating characteristics of the X2 change from single-channel mode to dual-channel mode by utilizing a traditional array of far-field diagnostics, which include a nude Faraday probe, retarding potential analyzer (RPA), and $\mathrm{E} \times \mathrm{B}$ probe. To the knowledge of the authors, measurements using these diagnostics have never been taken and reported for a nested-channel Hall thruster.

Data collected from these probes are used to determine five efficiencies that are part of existing phenomenological models for Hall thrusters. ${ }^{5,6}$ The five efficiencies are:

- Current utilization efficiency, $\eta_{b}$

- Divergence efficiency, $\eta_{d}$

- Charge utilization efficiency, $\eta_{q}$

- Voltage utilization efficiency, $\eta_{v}$

- Total mass utilization efficiency, $\eta_{M}$

The total efficiency, $\eta_{t}$, of a Hall thruster can be broken down into various contributing efficiencies by the following equation:

$$
\eta_{t}=\frac{T^{2}}{2 \dot{m}_{t} P_{t}}=\eta_{b} \eta_{d} \eta_{q} \eta_{v} \eta_{M} \eta_{m a g}
$$

where the electromagnet efficiency, $\eta_{\text {mag }}$, is the ratio of the discharge power to the total power, $P_{d} / P_{t}$.

Current density profiles from a Faraday probe were used to calculate divergence efficiency, current utilization efficiency, and total mass utilization efficiency. The current fractions from the measured spectra of the E $\times \mathrm{B}$ probe were used to determine charge utilization efficiency and total mass utilization efficiency. Measurements of the RPA and an adjacent Langmuir probe (LP) were used to determined voltage utilization efficiency. The discharge power and total power were directly measured by a data acquisition system and shunt resistors.

\section{Methodology for Calculation of Parameters}

\section{A. Current Utilization}

$$
\begin{gathered}
\eta_{b}=\frac{I_{b}}{I_{d}} \\
I_{b}=2 \pi R^{2} \int_{0}^{\frac{\pi}{2}} \frac{I(\theta, R)}{A_{c}+\kappa_{G}} \frac{\kappa_{D}\left(\theta, R, R_{C L_{1}}, R_{C L_{2}}\right)}{\kappa_{A}\left(\theta, R, R_{C L_{1}}, R_{C L_{2}}\right)} \sin (\theta) d \theta
\end{gathered}
$$

$\eta_{b}$ is the ratio of the beam current to the discharge current, as shown in Equation 2, and is a measure of how much of the input current is carried by thrust-producing ions. Current collected by the Faraday probe, $I(\theta, R)$, and discharge current, $I_{d}$, are determined by measuring the voltages across shunt resistors. Beam current, $I_{b}$, is calculated by hemispherical integration of the azimuthal distribution of ion current density as measured by a Faraday probe. The full expression for $I_{b}$ is shown in Equation 3, where the angular position, $\theta$, is centered about thruster centerline. Corrections for the collection area, $A_{c}$, and the radial position, $R$, were implemented in this study to improve the accuracy of the nude Faraday probe measurements, as described by Brown and Gallimore. ${ }^{7,8}$

$\kappa_{G}$ is the gap correction factor and accounts for ion current collection along the side-walls of the Faraday probe collector. The area correction factor, $\kappa_{A}$, corrects for the cosine losses due to the annular geometry of the ion source. The distance correction factor, $\kappa_{D}$, corrects for the error in radial distance $R$ from the annular ion source, as $R$ is measured with respect to thruster centerline and not with respect to the channels. However, the original formulations of $\kappa_{A}$ and $\kappa_{D}$ were meant for a single-channel Hall thrusters. For use with the X2 in dual-channel mode, the correction factors were modified to include both annular ion sources, as shown in Equations 4 and 5.

$$
\begin{aligned}
\kappa_{A} & =\cos ^{2}\left[\frac{\sum_{i=1}^{N}\left(\alpha_{L_{i}}+\alpha_{R_{i}}\right) I_{d_{i}}}{2 \sum_{i=1}^{N} I_{d_{i}}}\right] \\
\alpha_{L_{i}, R_{i}} & =\mp \theta \mp \tan ^{-1}\left(\frac{-\sin (\theta)-\frac{R_{C L_{i}}}{R}}{\cos (\theta)}\right)
\end{aligned}
$$


$\alpha_{L_{i}, R_{i}}$ are angles of incidence from the left and right centerlines, respectively, of the $i$ th of $N$ annuli in the measurement plane of the Faraday probe. For the $\mathrm{X} 2, N$ is 2 . The angles of incidence to each channel are weighted by the corresponding $I_{d_{i}}$ such that $\kappa_{A}$ is biased toward the channel that dominates $I_{b}$. If the current from one of two channels goes to zero, the single-channel $\kappa_{A}$ is recovered for the remaining channel. This allows $\kappa_{A}$ to be remain valid regardless of X2's operating mode. Discharge currents are used in the weighting instead of beam currents because far-field Faraday probe measurements are unable to distinguish between the individual ion beams.

$$
\begin{gathered}
\kappa_{D}=\left[\frac{\sum_{i=1}^{N}\left(\frac{R_{L_{i}}}{R}+\frac{R_{R_{i}}}{R}\right) I_{d_{i}}}{2 \sum_{i=1}^{2} I_{d_{i}}}\right] \\
\frac{R_{L_{i}, R_{i}}}{R}=\sqrt{[\cos (\theta)]^{2}+\left[-\sin (\theta) \mp \frac{R_{C L_{i}}}{R}\right]^{2}}
\end{gathered}
$$

As shown in Equations 6 and 7, $\kappa_{D}$ was similarly modified to include both channels. The normalized distances $R_{L_{i}, R_{i}} / R$ are also weighted by the corresponding discharge current, biasing the $\kappa_{D}$ toward its value for the dominant channel. For the X2 in single-channel mode, the original formulation $\kappa_{D}$ is recovered for the one active channel.

\section{B. Divergence}

$$
\begin{gathered}
\eta_{d}=[\cos (\beta)]^{2}=\left(\frac{I_{\text {axial }}}{I_{b}}\right)^{2} \\
\beta=\cos ^{-1}\left(\frac{I_{\text {axial }}}{I_{b}}\right) \\
I_{\text {axial }}=2 \pi R^{2} \int_{0}^{\frac{\pi}{2}} \frac{I(\theta, R)}{A_{c}+\kappa_{G}} \frac{\kappa_{D}\left(\theta, R, R_{C L_{1}}, R_{C L_{2}}\right)}{\kappa_{A}\left(\theta, R, R_{C L_{1}}, R_{C L_{2}}\right)} \cos (\theta) \sin (\theta) d \theta
\end{gathered}
$$

The parameter $\eta_{d}$ describes the degree of collimation in the ion beam from the Hall thruster and is related to the plume divergence half-angle $\beta$ by Equation $8 .^{6} \beta$ is calculated by using the ratio of axial beam current, $I_{\text {axial }}$, to total beam current, $I_{b}$, as shown in Equation 9. As expressed in Equation 10, $I_{\text {axial }}$ is determined in a manner similar to the calculation of $I_{b}$ with an addition factor of $\cos (\theta)$ to account for the orientation of the probe.

\section{Charge Utilization}

$$
\eta_{q}=\frac{\left(\sum_{i=1}^{n} \frac{\Omega_{i}}{\sqrt{Z_{i}}}\right)^{2}}{\sum_{i}^{n} \frac{\Omega_{i}}{Z_{i}}}
$$

Charge utilization efficiency $\eta_{q}$, as defined in Equation 11, describes the composition of the plume in terms of the charge states of the ions. $\eta_{q}$ is unity if all ions are singly-charged and becomes less than unity when neutrals become multiply-charged.

The current fraction of the $i$ th ion species, $\Omega_{i}$, is determined from an $\mathrm{E} \times \mathrm{B}$ probe trace by the methods outlined by Shastry et al. ${ }^{9}$ Specifically, the triangle method and simplified charge exchange model are used in this study to calculate $\Omega_{i}$. $Z_{i}$ is the ion charge state. For the purposes of this study, values for $Z_{i}$ greater than 4 are not considered, as quadruply-charged current fractions are already very small and on the order of the associated uncertainty.

\section{Voltage Utilization}

$$
\eta_{v}=\frac{V_{a}}{V_{d}}=\frac{V_{m p}-V_{p}}{V_{d}}
$$

Voltage utilization efficiency, $\eta_{v}$, is the fraction of the discharge voltage used for acceleration of ions, as described in Equation 12. The accelerating voltage, $V_{a}$, is determined by methods outlined by Hofer. ${ }^{10}$ RPA traces are numerically differentiated and the voltage corresponding to the maximum in the negative of the differentiated signal is known as the most-probable voltage, $V_{m p}$. Using an adjacent Langmuir probe, the local plasma potential, $V_{P}$, is determined by finding the voltage of the characteristic "knee" in the I-V characteristic. As shown in Equation 12, the most-probable voltage is corrected by the plasma potential to calculate the accelerating voltage. 


\section{E. Mass Utilization}

$$
\begin{gathered}
\eta_{M}=\eta_{m} \eta_{c}=\frac{m_{X e} I_{d}}{\dot{m}_{t} e} \eta_{b} \sum_{i=1}^{n} \frac{\Omega_{i}}{Z_{i}}=\frac{m_{X e} I_{b}}{\dot{m}_{t} e Q} \\
\eta_{m}=\frac{m_{X e} I_{d}}{\dot{m}_{a} e} \eta_{b} \sum_{i=1}^{n} \frac{\Omega_{i}}{Z_{i}}=\frac{m_{X e} I_{b}}{\dot{m}_{a} e Q} \\
\eta_{c}=\frac{\dot{m}_{a}}{m_{t}}
\end{gathered}
$$

Total mass utilization efficiency is the fraction of the supplied propellant mass flow rate that is converted into thrust-producing ions, as described in Equation 13. $\eta_{M}$ is the only parameter that is not directly measured by a probe but uses FP and $\mathrm{E} \times \mathrm{B}$ measurements to estimate the ion mass flow rate. The ion beam current, $I_{b}=I_{d} \eta_{b}$, is converted into mass flow rate by the mass-to-average charge ratio of ions, $m_{X e} / e Q$.

It should be noted that the definition of $\eta_{M}$ used in Equation 13 is from work performed by Brown et al and combines the mass utilization, $\eta_{m}$, and cathode efficiency, $\eta_{c}$, from Hofer et al. ${ }^{5,6}$ However, the definition of total efficiency, $\eta_{t}$, remains consistent. The expressions for $\eta_{m}$ and $\eta_{c}$ are shown in Equations 14 and 15, respectively.

\section{Experimental Apparatus}

\section{A. Nested-Channel Hall-Effect Thruster}

The X2 NHT features two discharge channels with similar cross-sectional dimensions, magnetic field topologies, and gas distributors. During experimentation, the radial magnetic field pointed radially inward across the inner channel and radially outward across the outer channel. Testing of the X2 was conducted with an updated version of the internallymounted $\mathrm{LaB}_{6}$ hollow cathode used for a 6-kW laboratory Hall thruster. At the beginning of every pumpdown, both channels of the $\mathrm{X} 2$ were operated for a bake-out period of one hour.

Both anodes and the cathode had separate stainless steel propellant feed lines with viton isolators at the thruster. MKS Model 1179A mass flow controllers were used to regulate propellant flow through the outer anode and the cathode, and an MKS Model 1159B mass flow controller was used for the inner anode. All three mass flow controllers were connected to an MKS Model 247C 4-channel readout. The manufacturer-specified accuracy of the mass flow controllers is $1 \%$ of full scale. Research grade xenon at $99.999 \%$ purity was used throughout testing.

Electrical connections for the X2 were made such that each channel had a separate discharge power supply, and the common for both discharge circuits was connected to the shared internal cathode. This electrical configuration was based on previous work performed at PEPL on clustered Hall thrusters with a shared hollow cathode. ${ }^{11,12}$ The thruster was electrically grounded to the facility ground during testing, and discharge voltages were measured from contacts at the back of the X2.

The inner channel discharge was powered by a Sorensen PRO 600-16T while the outer channel discharge was powered by an American Reliance HPS 1000-100-K027. Power for the magnets were provided by three EMI EMS 60-10 power supplies and one EMI EMS 100-10. The two trim coils were powered by a Sorensen DCS 60-18E and a DCS 600-1.7. Cathode keeper power was provided by an EMI EMS 600-1.6, and heater power was provided by a Sorensen DCS 33-33.

\section{B. Faraday Probe}

The nude Faraday probe used in this study was a nested Faraday probe based on prior work by Brown and Gallimore. ${ }^{7,8}$ The collectors and guard ring were made from graphite and the ceramic shell was composed of macor. Graphite was chosen for its low secondary electron yield when bombarded with xenon, and both graphite and macor were selected for their machinability. The current collected by the inner and outer collectors were determined independently by measuring the voltages across shunt resistors with resistances of $107.1 \Omega$ and 107.4 $\Omega$, respectively. Both collectors were biased to $-30 \pm 0.1 \mathrm{~V}$ with respect to facility ground by a Kikusui PAD 55-6L power supply. This voltage was found to place the collectors well within ion saturation in the plume of the X2.

The Faraday probe was swept at a radius of $1 \mathrm{~m}$ from the center of the exit plane of the $\mathrm{X} 2$, as illustrated in Figure 3(b). This distance for $R$ was chosen for its ubiquity in Hall thruster Faraday probe measurements. The coordinate system for the Faraday probe traces places the origin at the exit plane along thruster centerline so $\theta$ is zero along the thrust axis. 


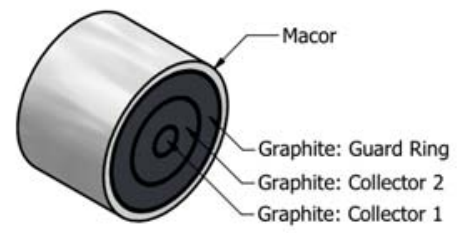

(a) Nested Faraday probe

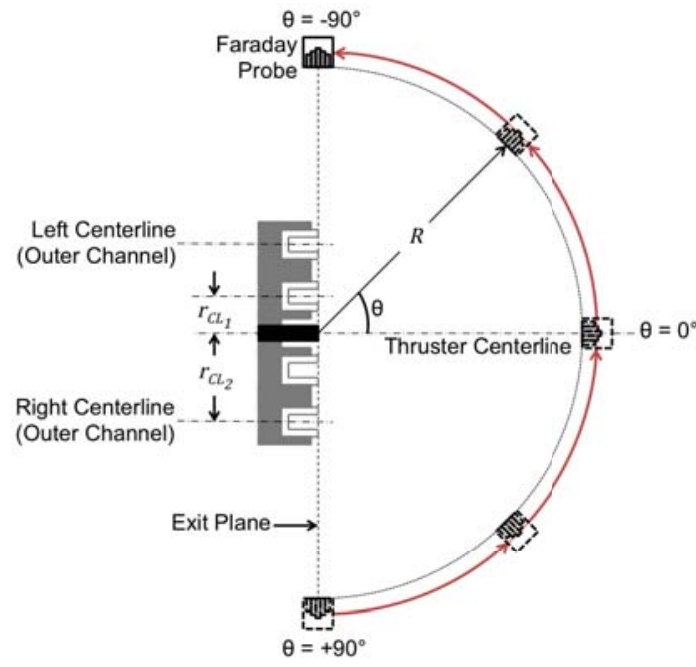

(b) Faraday probe actuation

Figure 3: Nested Faraday probe and top view of the actuation of the Faraday probe

\section{C. $\quad$ E $\times$ B Probe}

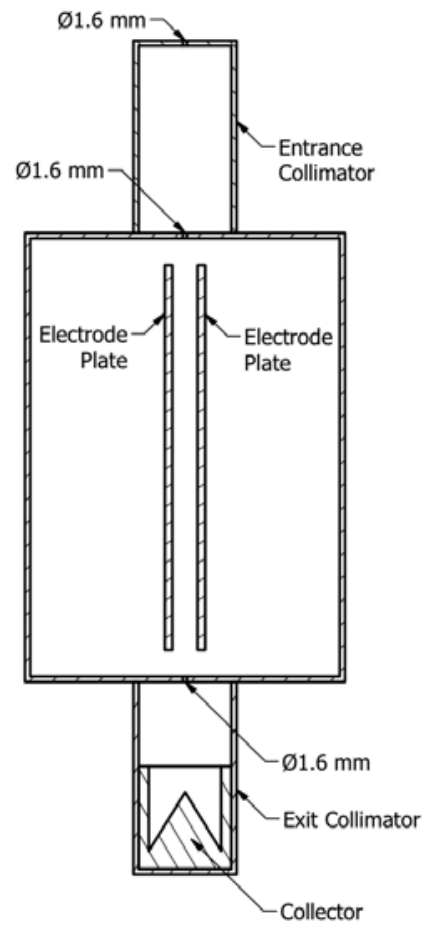

(a) $\mathrm{E} \times \mathrm{B}$ probe schematic (Not to scale)

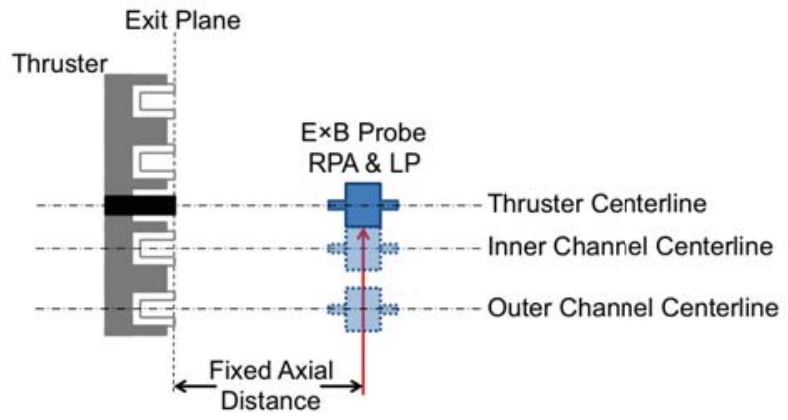

(b) $\mathrm{E} \times \mathrm{B}$ probe, RPA, and LP actuation

Figure 4: $E \times B$ probe schematic and top view of actuation of $E \times B$ probe, RPA, and LP

The E $\times \mathrm{B}$ probe used in this study was designed by NASA Glenn Research Center and is more thoroughly described in previous work by Reid et al. ${ }^{13}$ The probe features a 150 -mm test section with 75 -mm entrance and exit collimators. Aperture diameters for the entrance orifice and the collimating orifices were all approximately $1.6 \mathrm{~mm}$. The internal magnetic field was created by sintered hard ferrite permanent magnets, while the internal electric field was generated by a Keithley 2410 Sourcemeter via two parallel conducting plates. Ions that passed through the test section were 
collected by a cone-and-cylinder collector that has been spray coated with tungsten to reduce secondary electron emission. Collected current was measured by a Keithley 6485 Picoammeter.

\section{Retarding Potential Analyzer}

The RPA used in this study was originally designed and built by the Air Force Research Laboratory and is described in further detail in separate documents by Reid and Hofer. ${ }^{10,14}$ As shown in Figure 5, the grids and the collector of the RPA are separated by macor spacers which are housed within a macor sleeve and stainless steel shell. In order to repel electrons, the electron repelling grid was biased to $-30 \pm 0.1 \mathrm{~V}$ with respect to facility ground by an HP Model $721 \mathrm{~A}$ power supply. The voltage applied to the ion retarding grid was swept relative to facility ground by a Keithley 2410 Sourcemeter, and the collected current was measured by a Keithley 6485 Picoammeter.

A cylindrical Langmuir probe was placed adjacent to the RPA for the necessary measurements of local plasma potential. The Langmuir probe was composed of a $0.25-\mathrm{mm}$ diameter tungsten wire that protruded $6.6 \pm 0.1 \mathrm{~mm}$ from a 1.5 -mm diameter alumina tube.

The $\mathrm{E} \times \mathrm{B}$ probe, $\mathrm{RPA}$, and Langmuir probe were placed $2.1 \mathrm{~m}$ downstream of the exit plane to minimize probe heating and perturbations to the thruster. The $\mathrm{E} \times \mathrm{B}$ probe was protected by graphite plates in front and on the plume side to further prevent overheating of the probe body. The RPA was protected by a graphite shutter and the shadow of the $\mathrm{E} \times \mathrm{B}$ probe when the probe array was not in use. Radial actuation was provided by a $1.5-\mathrm{m}$ linear translation stage, allowing each of probes to be placed directly downstream of the individual centerlines of the inner channel and outer channel. The axial and lateral

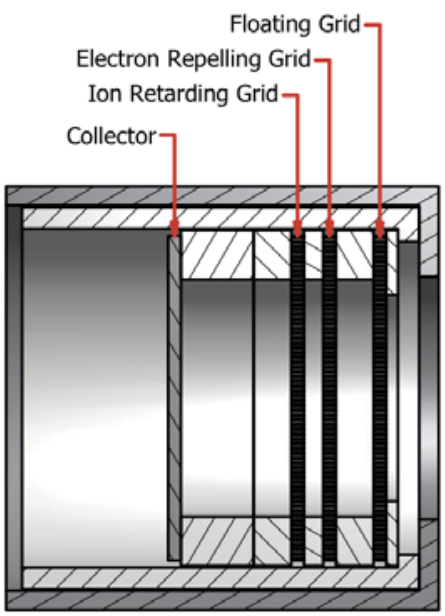
position uncertainties were both approximately $\pm 1 \mathrm{~mm}$.

\section{E. Vacuum Facility}

Experiments on the X2 were performed in the Large Vacuum Test Facility (LVTF) in PEPL at the University of Michigan. LVTF is a cylindrical vacuum chamber that measures 6 meters in diameter and 9 meters in length. Seven CVI model TM-1200 cryopumps with liquid nitrogen shrouds are used to achieve a base pressure of $3 \times 10^{-7}$ torr. The nominal pumping speed for xenon is $245,000 \mathrm{l} / \mathrm{s}$ with all seven cryopumps operating. Pressure was measured using a Varian UHV-24 nude ionization gauge and a Varian model 571 Bayard-Alpert ionization gauge connected to a Varian XGS-600 gauge controller. The reported background pressures are an average of the pressure measurements taken by the two gauges. Typical accuracy for ionization gauges is approximately $\pm 20 \%$ according to Varian ${ }^{15}$. The difference in pressure measurements between the two ionization gauges varied with operating condition between $2 \%$ and $22 \%$.

\section{Results}

Table 1 shows the single-channel and dual-channel operating conditions during which far-field measurements were taken. For each dual-channel condition, an inner-channel condition and an outer-channel condition were performed under the same applied magnetic field and cathode flow fraction. The single-channel operating conditions were meant to closely approximate how each channel operates during dual-channel mode, but the influence of the adjacent plasma was naturally missing. However, differences in plume properties between the two modes can reveal some information about the interaction between channels.

This set of operating conditions is centered at the dual-channel condition with the highest thrust-to-power ratio: $150 \mathrm{~V}$ and $75 \%$ nominal mass flow rate. Discharge voltage and mass flow rate were changed across this condition of interest. The discharge voltages applied to both channels were always the same during this study, and the current densities of the two channels were nearly equal. The cathode flow fraction was kept constant at $10 \%$, as this fraction was found to work well at lower flow rates and lower discharge voltages. 
Table 1: X2 Operating Conditions

\begin{tabular}{|c|c|c|c|c|}
\hline $\begin{array}{c}\text { Discharge } \\
\begin{array}{c}\text { Voltage } \\
{[\mathrm{V}]}\end{array}\end{array}$ & $\begin{array}{c}\text { Anode Mass Flow Rate } \\
\text { (Inner Channel) } \\
{[\mathrm{mg} / \mathrm{s}]}\end{array}$ & $\begin{array}{c}\text { Anode Mass Flow Rate } \\
\text { (Outer Channel) } \\
{[\mathrm{mg} / \mathrm{s}]}\end{array}$ & $\begin{array}{c}\text { Percent Nominal } \\
\text { Flow Rate of } \\
\text { Operating Mode }\end{array}$ & $\begin{array}{c}\text { Background } \\
\text { Pressure } \\
\text { [torr-Xe] }\end{array}$ \\
\hline 150 & 4.7 & 11.6 & $50 \%$ & $1.0 \times 10^{-5}$ \\
100 & 7.0 & 17.4 & $75 \%$ & $1.5 \times 10^{-5}$ \\
150 & 7.0 & 17.4 & $75 \%$ & $1.6 \times 10^{-5}$ \\
200 & 7.0 & 17.4 & $75 \%$ & $1.5 \times 10^{-5}$ \\
150 & 9.3 & - & $100 \%$ & $2.1 \times 10^{-5}$ \\
\hline 150 & 4.7 & - & $50 \%$ & $3.1 \times 10^{-6}$ \\
100 & 7.0 & - & $75 \%$ & $4.5 \times 10^{-6}$ \\
150 & 7.0 & - & $75 \%$ & $4.5 \times 10^{-6}$ \\
200 & 7.0 & 11.6 & $75 \%$ & $4.5 \times 10^{-6}$ \\
150 & 9.3 & 17.4 & $50 \%$ & $5.8 \times 10^{-6}$ \\
\hline 150 & - & 17.4 & $75 \%$ & $1.1 \times 10^{-6}$ \\
100 & - & 17.4 & $75 \%$ & $1.1 \times 10^{-5}$ \\
150 & - & 23.2 & $100 \%$ & $1.0 \times 10^{-5}$ \\
200 & - & & & $1.5 \times 10^{-5}$ \\
\hline 150 & - & - & \\
\hline
\end{tabular}

\section{A. Faraday Probe}

The angular current density profiles measured by the Faraday probe are shown in Figures 6(a)-7(c). Measurements from the inner and outer collectors were found to be nearly identical, so only the inner collector measurements are presented here. All profiles in Figures 6(a)-7(c) include the gap and geometric corrections as previously described.

The X2 current density profiles exhibit expected characteristics for low-voltage operation. The plume is less collimated at low discharge voltages, resulting in a more gradual falloff in current density from the core ( 0 deg) to the wings $( \pm 90 \mathrm{deg})$. As discharge voltage increases, plume collimation improves, and more of the beam current is carried near the core. The current density distributions are less sensitive to changes in propellant mass flow rate, as the profiles at fixed discharge voltage are similar in geometry but are vertically shifted due to differences in total beam current.

The outer channel appears to dominate the combined plume, as it carries over twice the mass flow and current as the inner channel. However, the inner channel has some influence over the combined current density profile. Most of the outer-channel profiles exhibit asymmetry about the thruster centerline, but the asymmetries become less pronounced in the dual-channel profile. This appears to be a blending effect caused by the presence of additional inner-channel ions in the core of the plume.

For reference, the superpositions of the single-channel current density profiles are included for comparison to the dual-channel current density profiles. The superpositions of single-channel profiles could potentially be used as approximations of the dual-channel profiles, but they are shown in Figures 6(a)-7(c) to consistently underestimate current density in the core while overestimating in the wings.

The calculated current utilization efficiencies from the Faraday probe measurements are shown in Figures 8(a) and 8(b). Overall, $\eta_{b}$ increases from $100 \mathrm{~V}$ to $200 \mathrm{~V}$ and remains relatively constant with increasing flow rate at $150 \mathrm{~V}$. Differences between single-channel and dual-channel values of $\eta_{b}$ are small and practically negligible when including the measurement uncertainty.

None of the current density profiles presented in this paper have been extrapolated to vacuum, as additional measurements at two additional background pressures for each operating condition are currently unavailable. As a result, the lack of correction for charge-exchange increases the uncertainty in the current density profiles and the parameters calculated from them. The dominant source of uncertainty for $\eta_{b}$ is the relative uncertainty in $I_{b}$, which is approximately $5 \%$ for current density profiles that are not vacuum profiles. The uncertainty in $I_{d_{i}}$ is estimated to be \pm 0.1 A. 


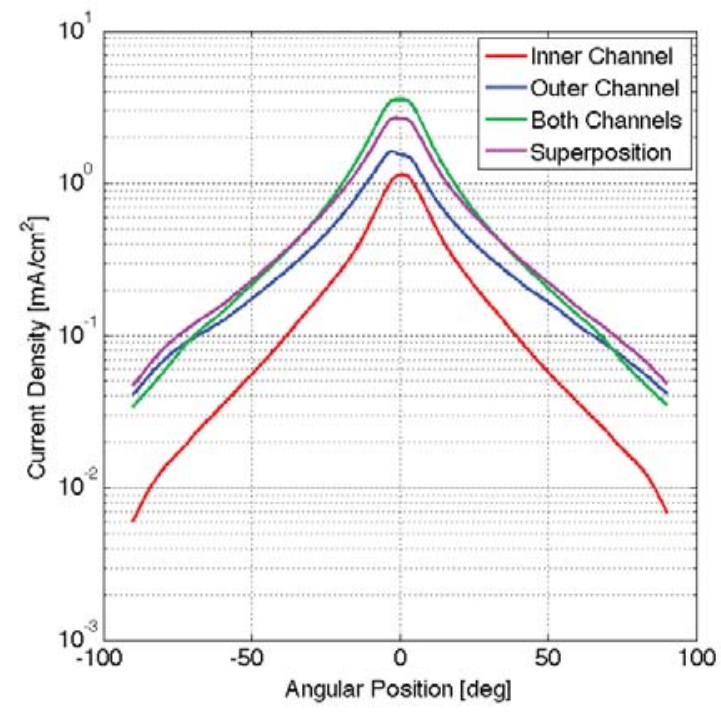

(a) Current density profiles at $100 \mathrm{~V}$

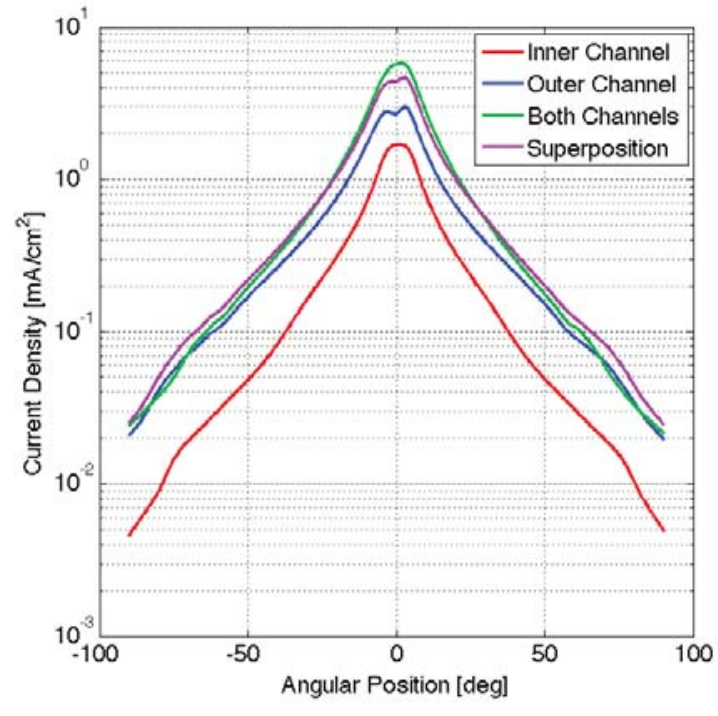

(b) Current density profiles at $150 \mathrm{~V}$

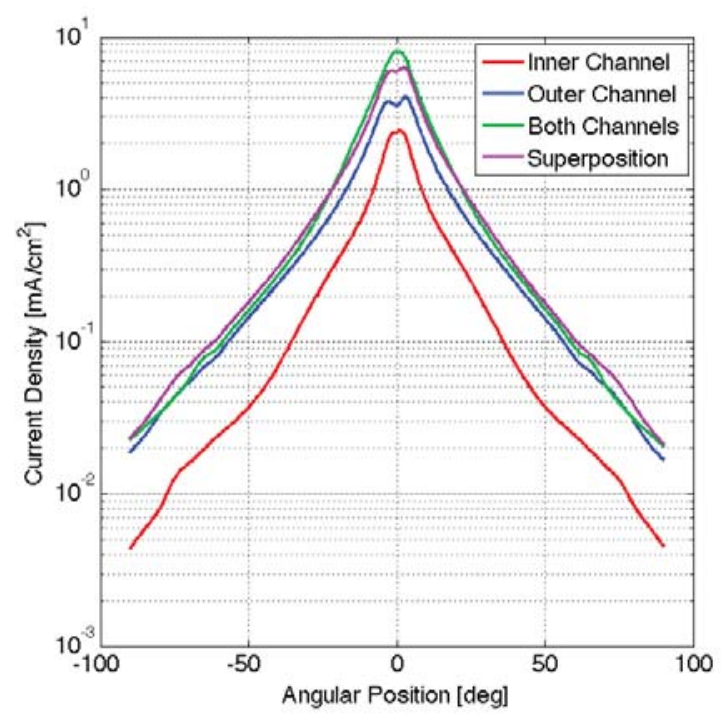

(c) Current density profiles at $200 \mathrm{~V}$

Figure 6: Single-channel and dual-channel current density profiles at fixed flow rate (75\% nominal) 


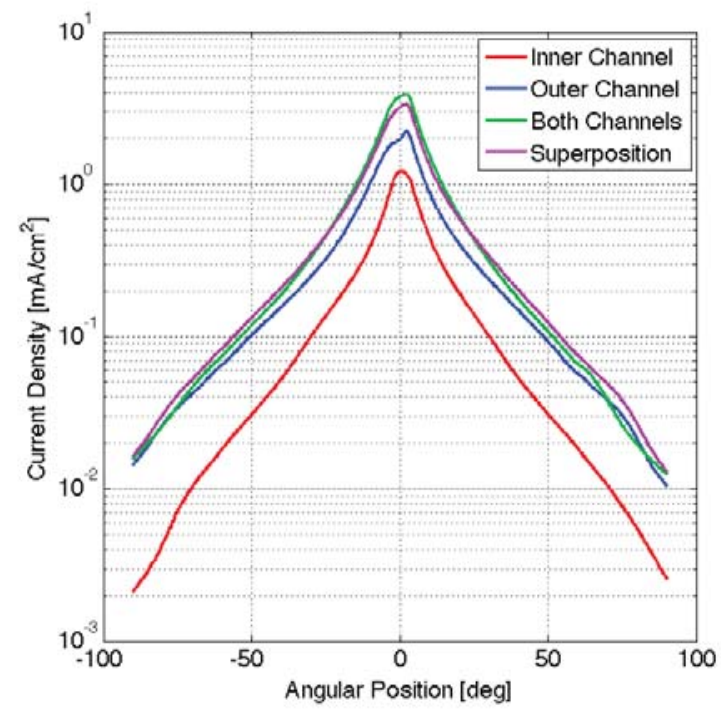

(a) Current density profiles at $50 \%$ nominal flow rate

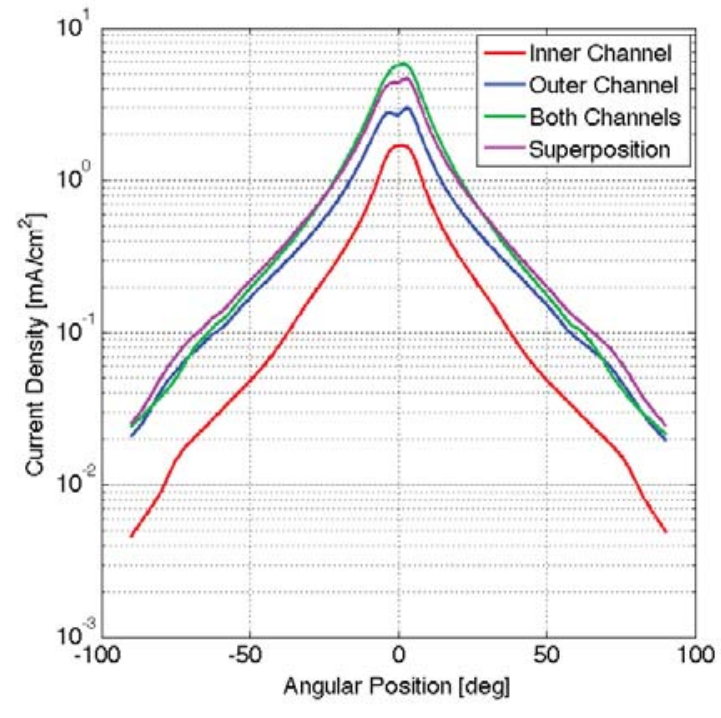

(b) Current density profiles at $75 \%$ nominal flow rate

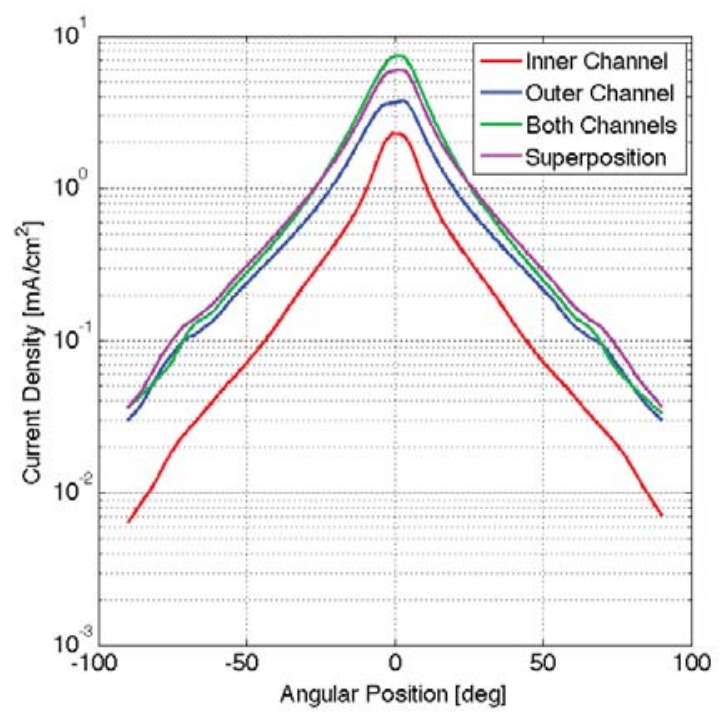

(c) Current density profiles at $100 \%$ nominal flow rate

Figure 7: Single-channel and dual-channel current density profiles at fixed discharge voltage (150 V) 
Divergence efficiency, as shown in Figures 9(a) and 9(b), also increases with discharge voltage and remains relatively constant with flow rate over their respective ranges. The uncertainty in divergence efficiency is relatively high because Faraday probe traces were not taken at multiple radial distances for each of the 15 operating conditions. As a result, the relative uncertainty in the ratio of $I_{\text {axial }}$ to $I_{b}$ increases from $5 \%$ to $10 \% .^{16}$

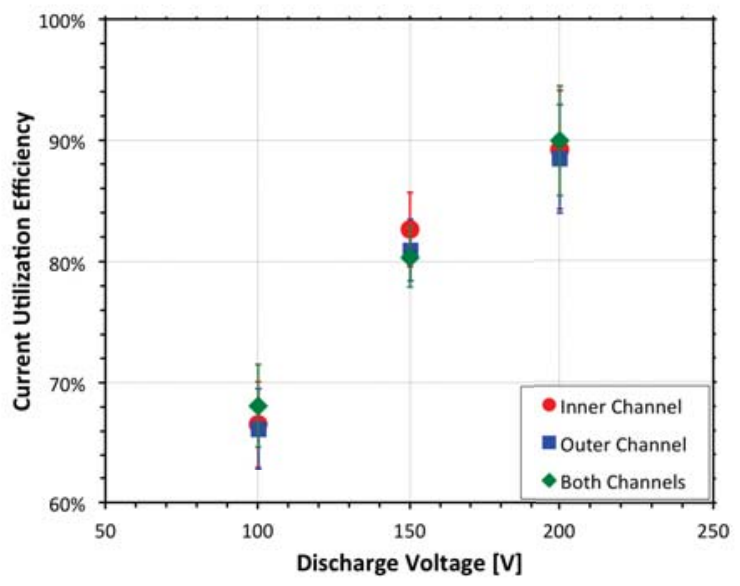

(a) Current utilization efficiency variation with voltage

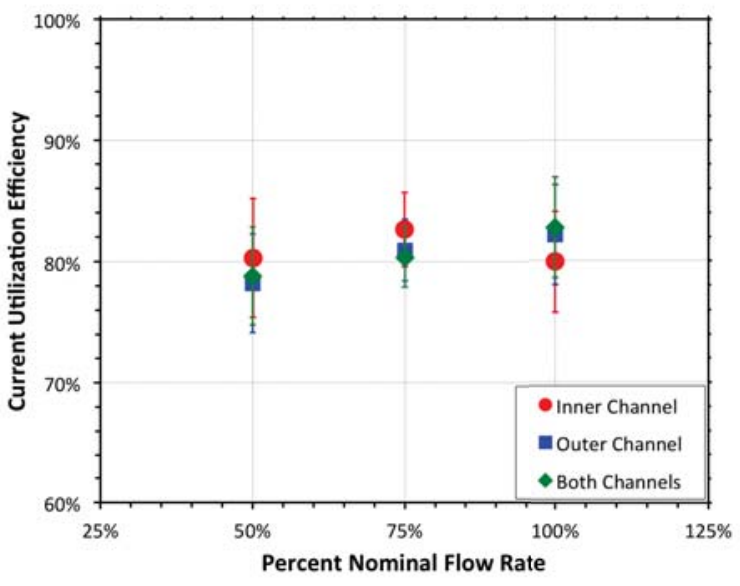

(b) Current utilization efficiency variation with mass flow rate

Figure 8: Current utilizations efficiencies

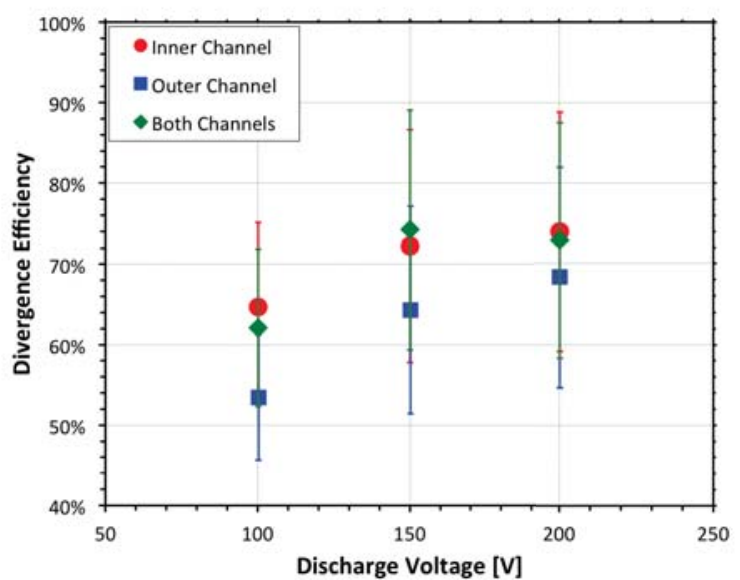

(a) Divergence efficiency variation with voltage

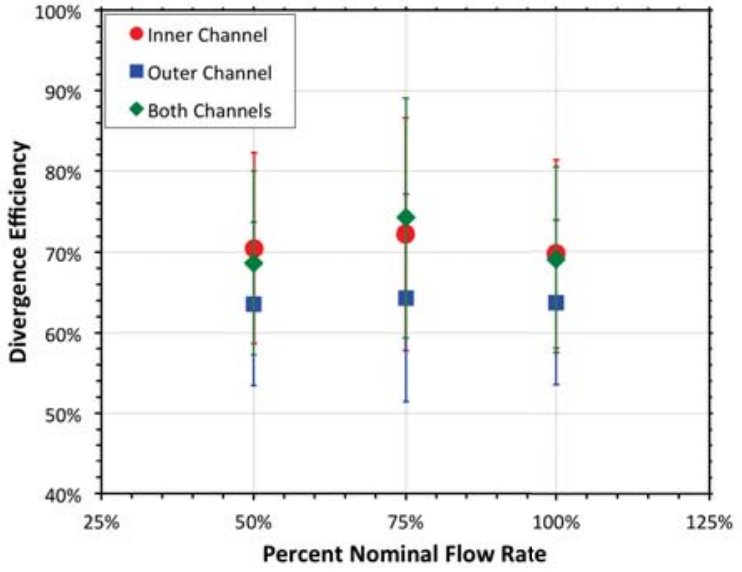

(b) Divergence efficiency variation with mass flow rate

Figure 9: Divergence efficiencies

\section{B. $\mathbf{E} \times \mathbf{B}$ Probe}

E $\times \mathrm{B}$ spectra for all operating conditions are shown in Figures 10(a) to 11(c). Each individual plot compares a singlechannel spectrum with a dual-channel spectrum using measurements that are most relevant to the single channel. All plots regarding to the inner channel has the $\mathrm{E} \times \mathrm{B}$ probe directly downstream of the inner channel, while all plots regarding the outer channel has the probe downstream of the outer channel.

The E $\times$ B spectra show expected trends with respect to discharge voltage and flow rate. Singly- and doubly-charged ions dominate at $100 \mathrm{~V}$, and increasing discharge voltage results in increasing current fractions of multiply-charged species. Increased flow rates also result in greater current fractions of multiply-charged species. Quadruply-charged xenon remained largely undetectable until $200 \mathrm{~V}$, where $\Omega_{4}$ was still approximately 0.01 .

Comparisons between dual-channel and single-channel spectra reveal some additional details regarding dualchannel operation. Firing both channels simultaneously inherently increases the total flow rate, so the current fractions 
of multiply-charge species increase during dual-channel operation as they do with increasing flow rate during singlechannel operation. Relative to the corresponding single-channel spectra, most of the dual-channel spectra are also shifted to higher applied voltages. This shift in the peaks of all charge species suggests that the ions arriving from the thruster are moving faster during dual-channel operation.

Charge utilization efficiencies are shown in Figures 12(a) and 12(b). The calculated values for $\eta_{q}$ are typical for Hall thrusters and do not vary significantly with discharge voltage or flow rate. Due to the increase in multiply-charged species during dual-channel mode, $\eta_{q}$ is typically lower with both channels active, but the drop is still small and not very significant considering the measurement error. Based on prior work with $\mathrm{E} \times \mathrm{B}$ analysis, the relative uncertainty for current fractions is $3 \%$ for $\Omega_{1}$ and $20 \%$ for $\Omega_{2}$ and $\Omega_{3} .{ }^{9}$ When necessary, the $20 \%$ relative uncertainty was also applied to $\Omega_{4}$.

Total mass utilization efficiencies, as based on Faraday probe and $\mathrm{E} \times \mathrm{B}$ probe measurements, are shown in Figures 13(a) and 13(b). Generally, $\eta_{M}$ increased with both discharge voltage and flow rate. Differences in $\eta_{M}$ between the two operating modes do not appear to be very significant, especially when the compounded measurement error is considered. To improve the uncertainty in $\eta_{M}$, improved measurements of $I_{b}$ would be necessary.

\section{RPA}

Figures 14(a) to 15(c) show all RPA traces after processing in order to better indicate the calculated acceleration voltage. The collected currents were numerically differentiated with respect to the voltage of ion retarding grid, and the differentiated signal was subsequently smoothed to reduce noise. Using the plasma potential measured by the adjacent Langmuir probe, the differentiated RPA signal was shifted such that the peak was located at $V_{a}$. The RPA and Langmuir probe were actuated in the same manner as the $\mathrm{E} \times \mathrm{B}$ probe, so all plots of RPA traces show measurements taken in alignment with either the inner-channel centerline or outer-channel centerline.

The measured acceleration voltages predictably increased with discharge voltage and remained relatively insensitive to flow rate. The differences in measured values of $V_{a}$ between operating modes show comparable or higher acceleration with both channels active. The higher $V_{a}$ was particularly pronounced for the inner channel at the operating point of interest: $150 \mathrm{~V}$ and $75 \%$ nominal flow rate. For the majority of operating conditions, the difference in $V_{a}$ for the outer channel was relatively small.

The trends in voltage utilization, as shown in Figures 16(a) and 16(b), reflect the trends in $V_{a} . \eta_{v}$ increases with discharge voltage, remains relatively constant with flow rate, and increases with both channels active. The increase in $\eta_{v}$ is greatest between the inner-channel and dual-channel operating conditions except at $100 \mathrm{~V}$. Uncertainty in $\eta_{v}$ is dominated by the uncertainty in acceleration voltage and in plasma potential. Based on the uncertainty of the numerical methods and signal noise, the estimated uncertainty for $V_{a}$ ranged from $3 \mathrm{~V}$ to $5 \mathrm{~V}$ and the uncertainty for $V_{p}$ was approximately $2 \mathrm{~V}$. The uncertainty in $V_{d}$ is estimated at $\pm 0.25 \mathrm{~V}$. 

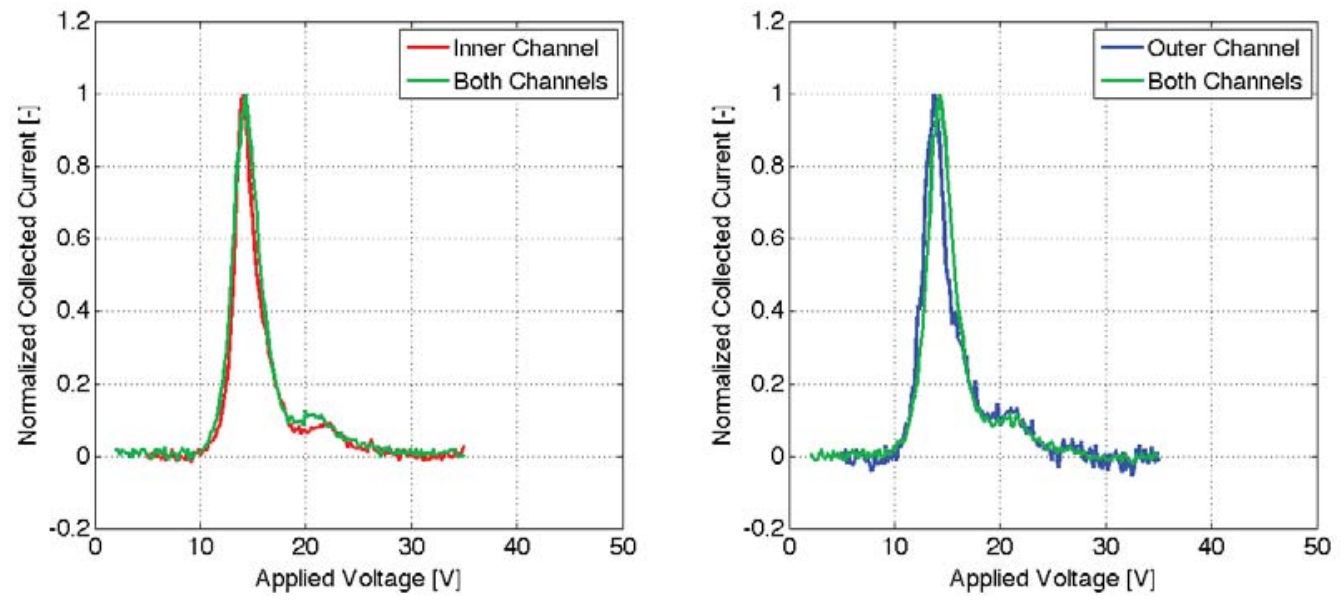

(a) $\mathrm{E} \times \mathrm{B}$ probe spectra at $100 \mathrm{~V}$
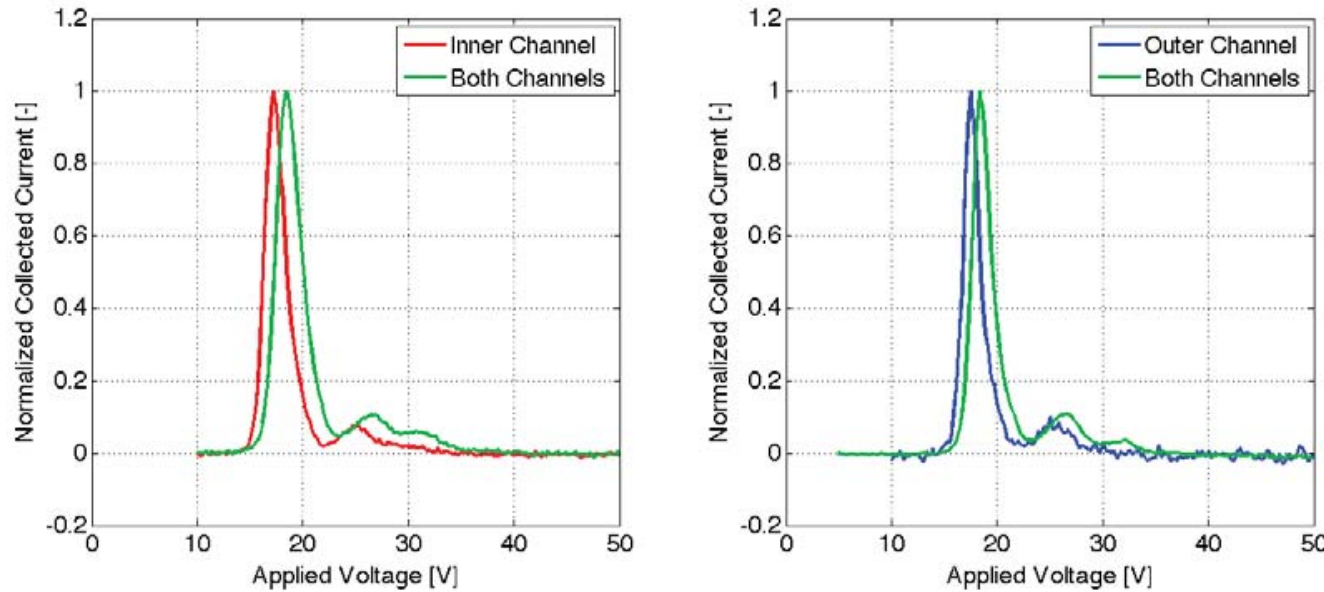

(b) $\mathrm{E} \times \mathrm{B}$ probe spectra at $150 \mathrm{~V}$
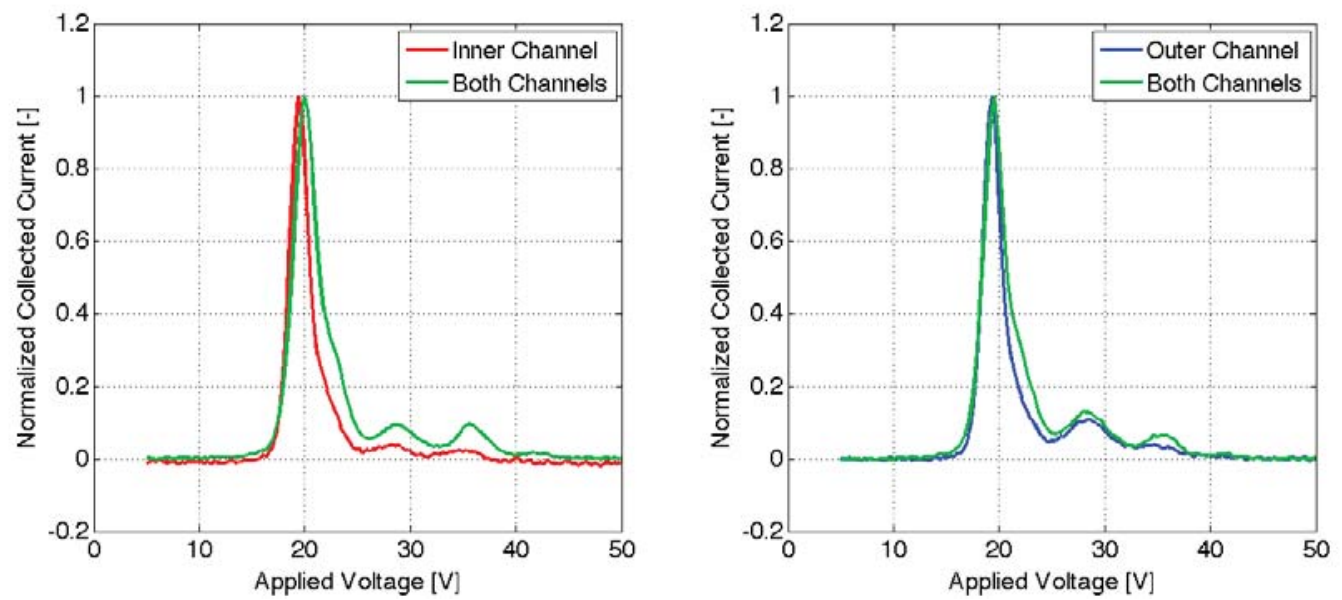

(c) $\mathrm{E} \times \mathrm{B}$ probe spectra at $200 \mathrm{~V}$

Figure 10: Single-channel and dual-channel $\mathbf{E} \times \mathbf{B}$ probe spectra at fixed flow rate (75\% nominal). Dual-channel spectra overlaid with inner-channel spectra were taken with the $\mathbf{E} \times \mathbf{B}$ probe aligned with the inner-channel centerline, while dual-channel spectra overlaid with outer-channel spectra were taken in alignment with the outer-channel centerline. 

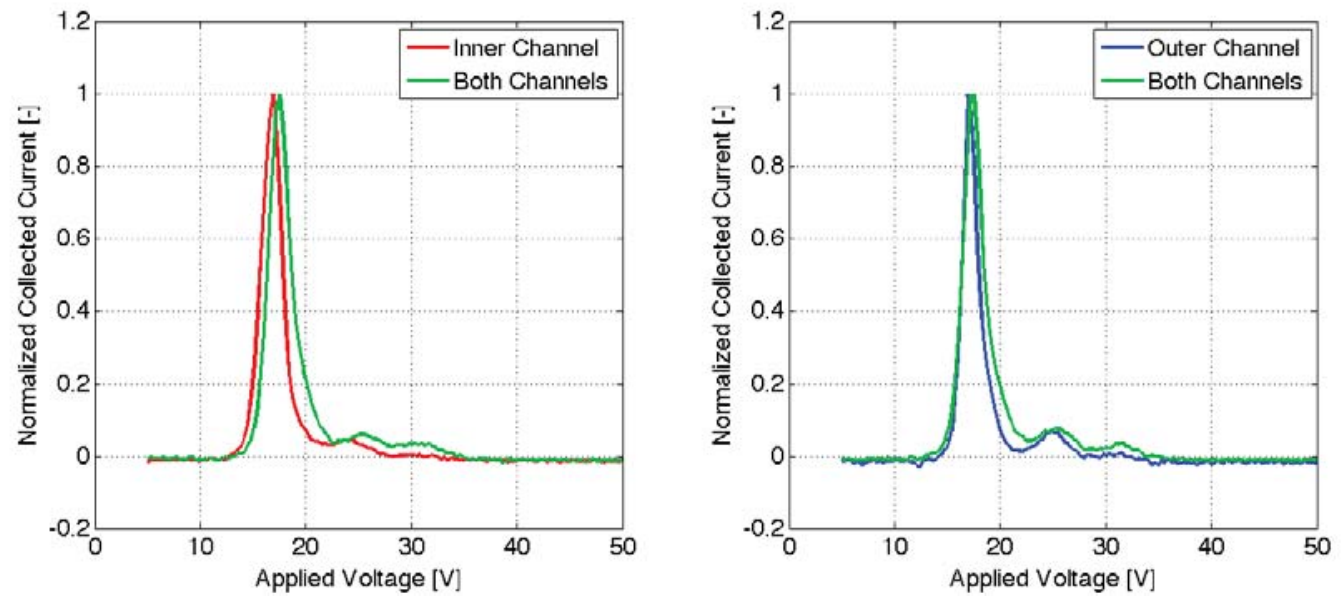

(a) $\mathrm{E} \times \mathrm{B}$ probe spectra at $50 \%$ nominal flow rate
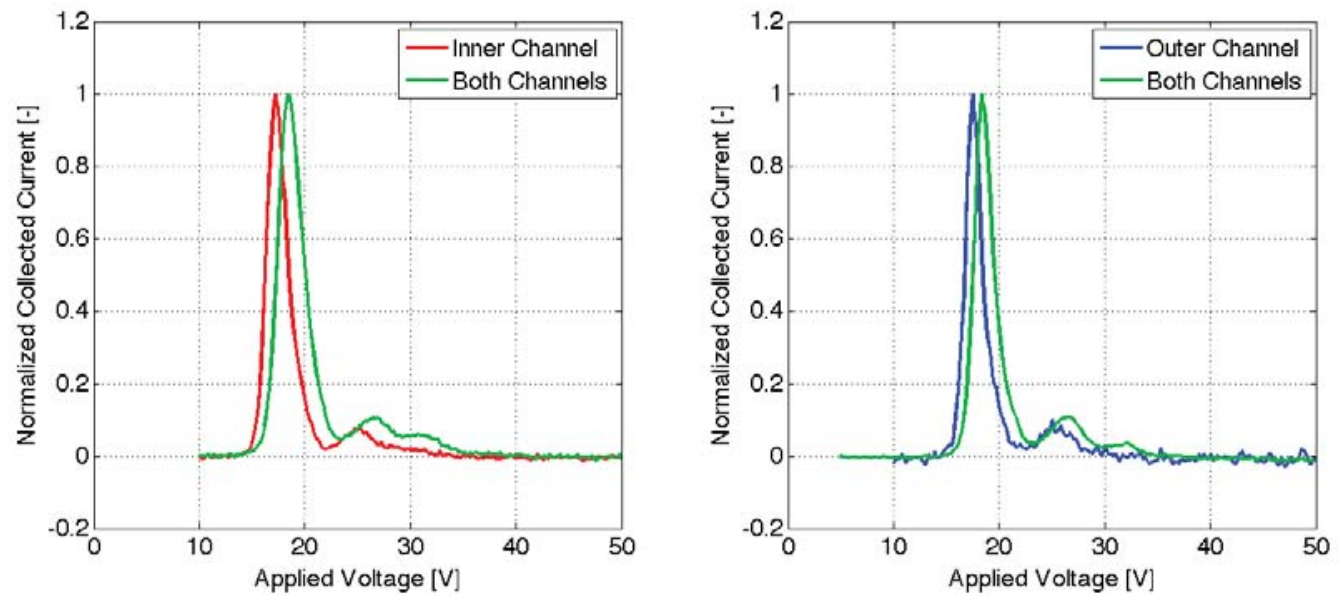

(b) $\mathrm{E} \times \mathrm{B}$ probe spectra at $75 \%$ nominal flow rate
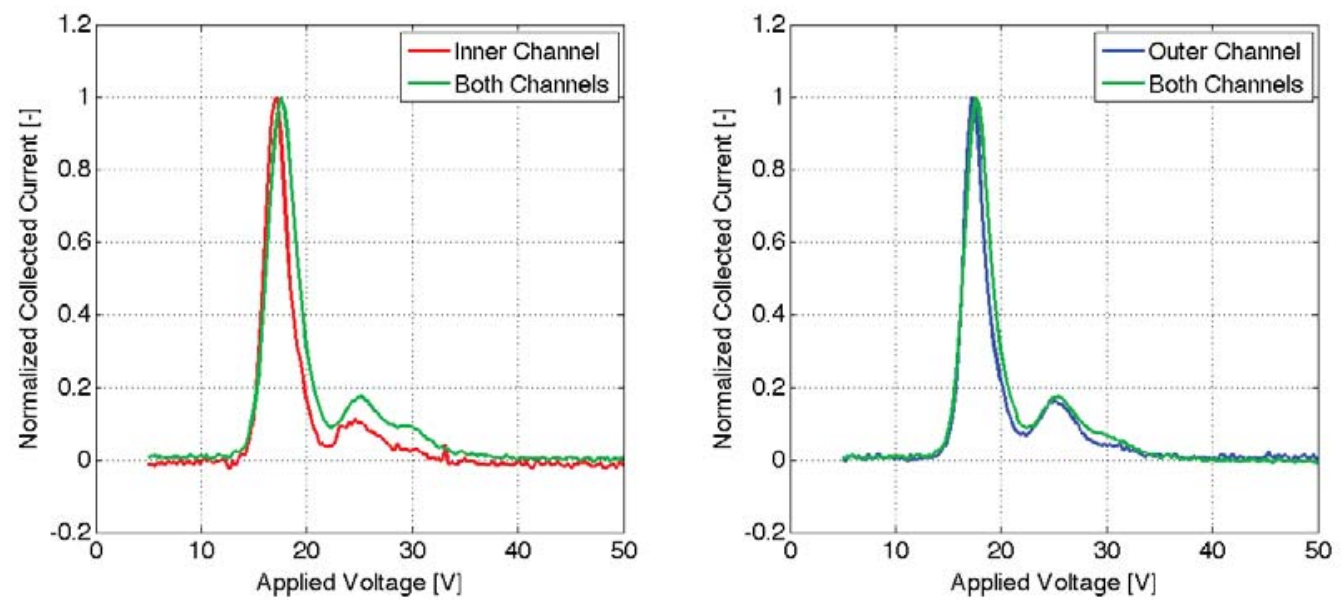

(c) $\mathrm{E} \times \mathrm{B}$ probe spectra at $100 \%$ nominal flow rate

Figure 11: Single-channel and dual-channel $\mathbf{E} \times \mathbf{B}$ probe spectra at fixed discharge voltage $(150 \mathrm{~V})$. Dual-channel spectra overlaid with inner-channel spectra were taken with the $\mathbf{E} \times \mathbf{B}$ probe aligned with the inner-channel centerline, while dual-channel spectra overlaid with outer-channel spectra were taken in alignment with the outer-channel centerline. 


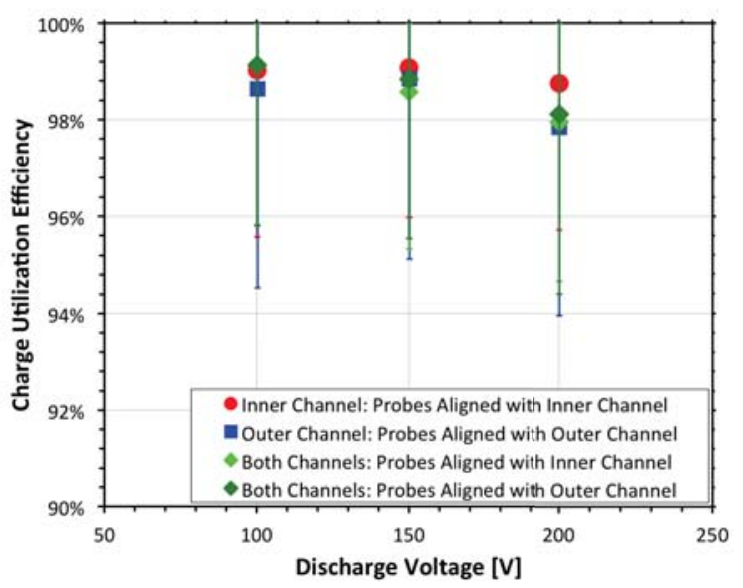

(a) Charge utilization efficiency variation with voltage

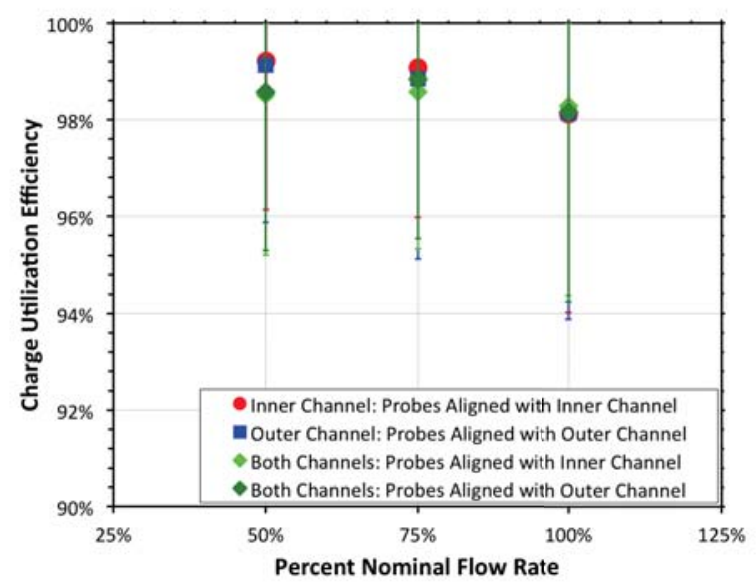

(b) Charge utilization efficiency variation with mass flow rate

Figure 12: Charge utilization efficiencies

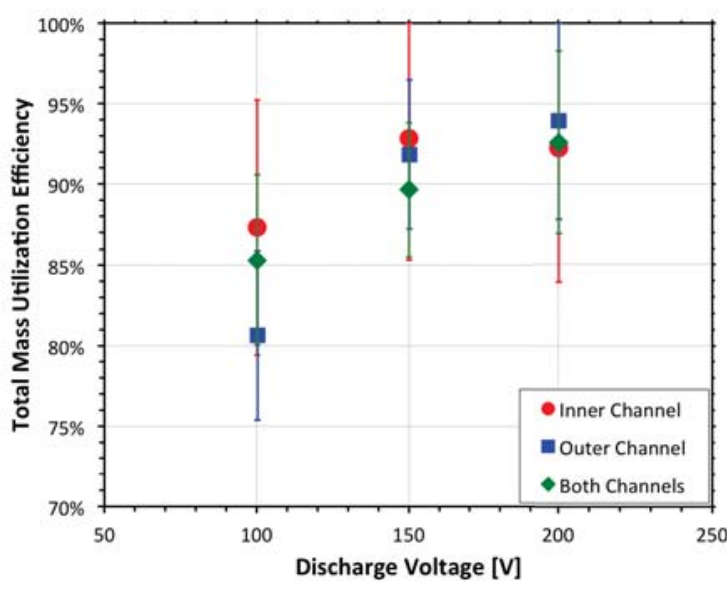

(a) Total mass utilization efficiency variation with voltage

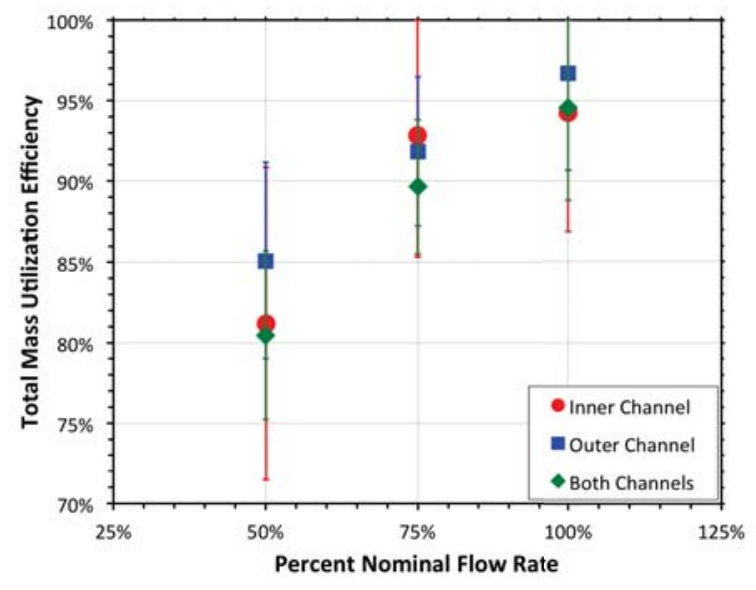

(b) Total mass utilization efficiency variation with mass flow rate

Figure 13: Total mass utilization efficiencies 

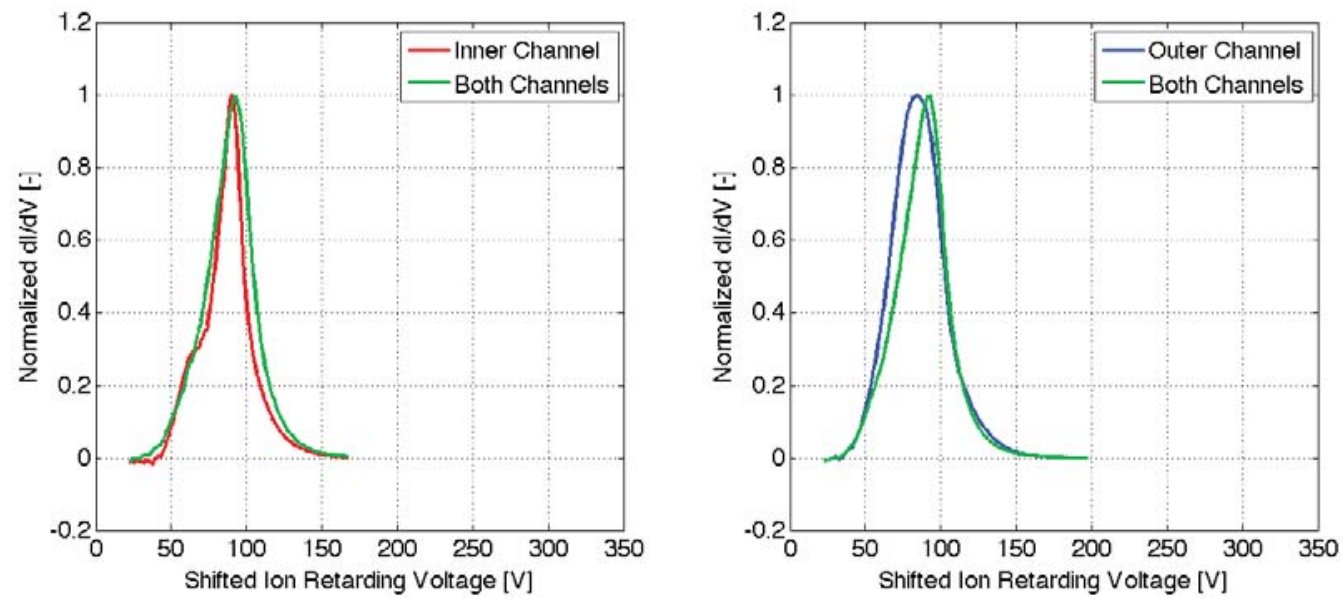

(a) Corrected and differentiated RPA traces at $100 \mathrm{~V}$
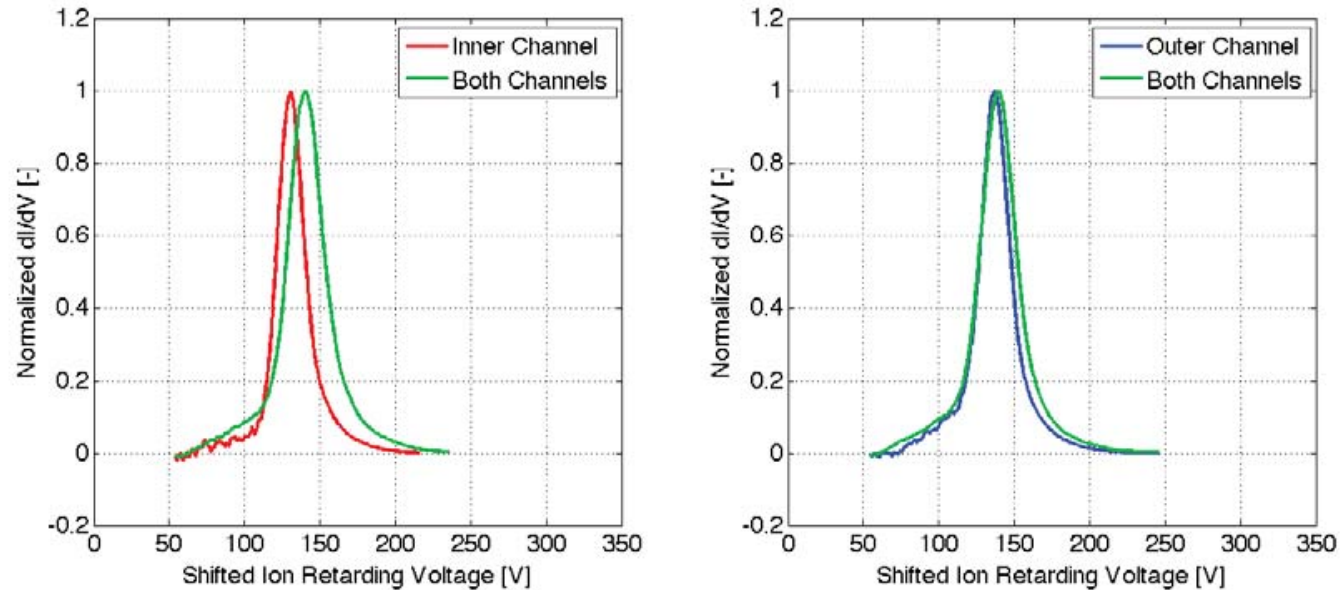

(b) Corrected and differentiated RPA traces at $150 \mathrm{~V}$
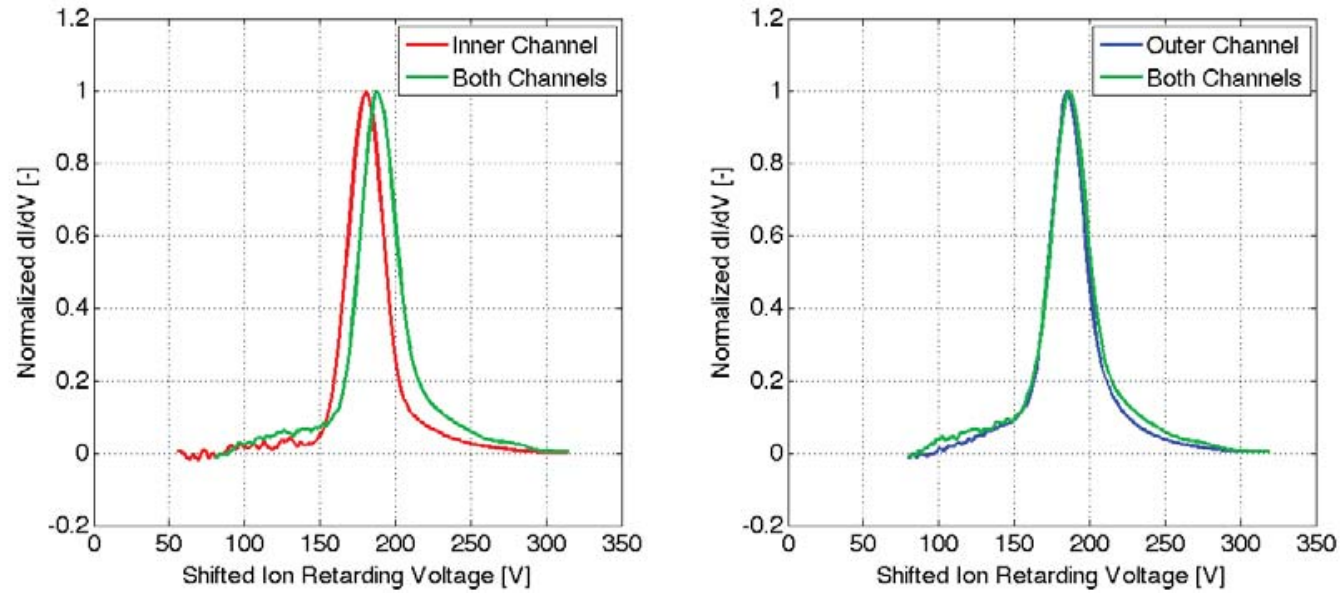

(c) Corrected and differentiated RPA traces at $200 \mathrm{~V}$

Figure 14: Single-channel and dual-channel differentiated RPA traces at fixed flow rate (75\% nominal). Ion retarding voltages were shifted by the corresponding plasma potentials to place the peaks at the accelerating voltages. Dual-channel spectra overlaid with inner-channel spectra were taken with the RPA and LP aligned with the inner-channel centerline, while dual-channel spectra overlaid with outer-channel spectra were taken in alignment with the outer-channel centerline. 

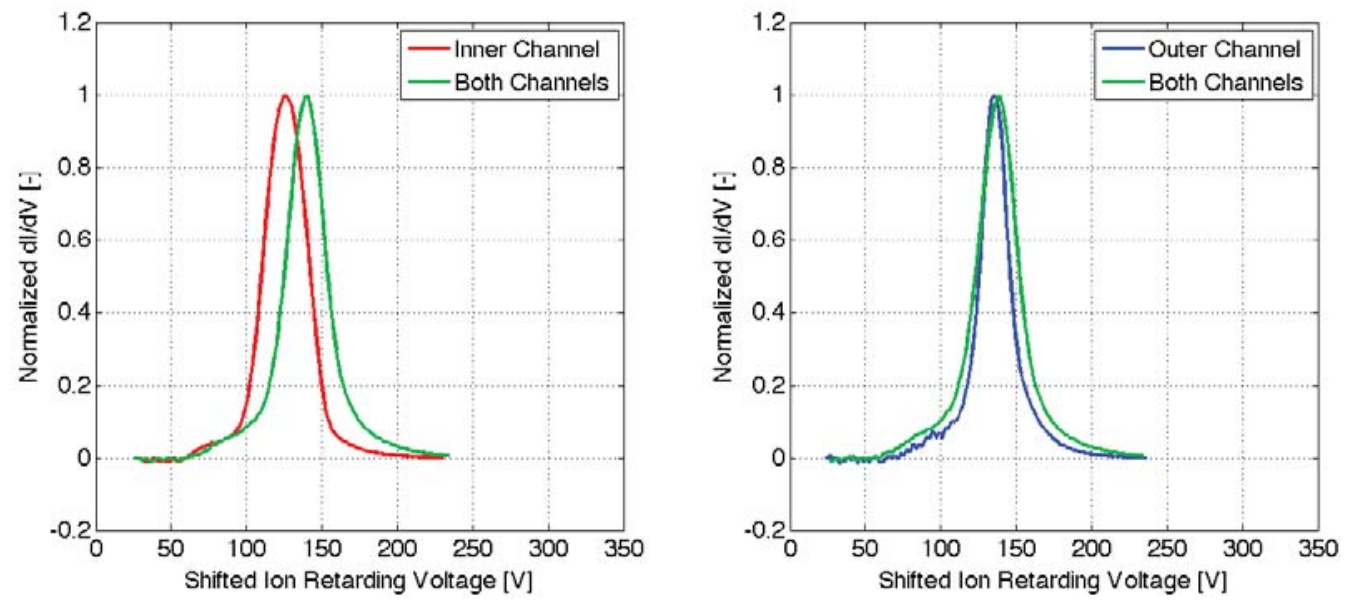

(a) Corrected and differentiated RPA traces at 50\% nominal flow rate
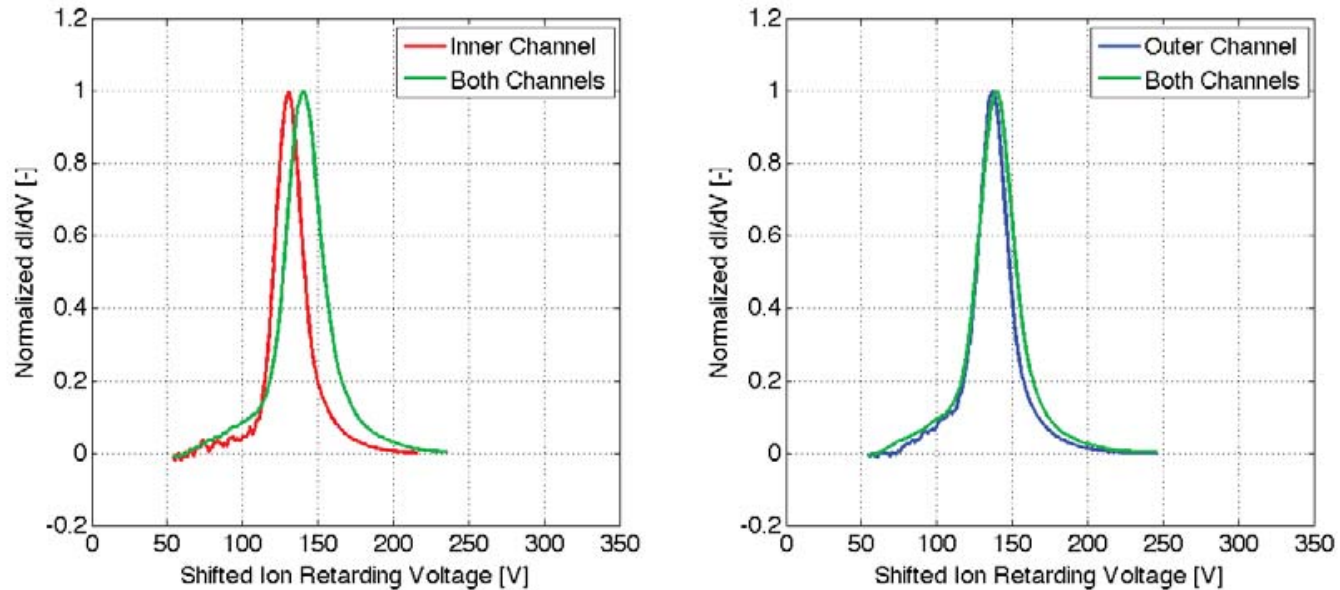

(b) Corrected and differentiated RPA traces at $75 \%$ nominal flow rate
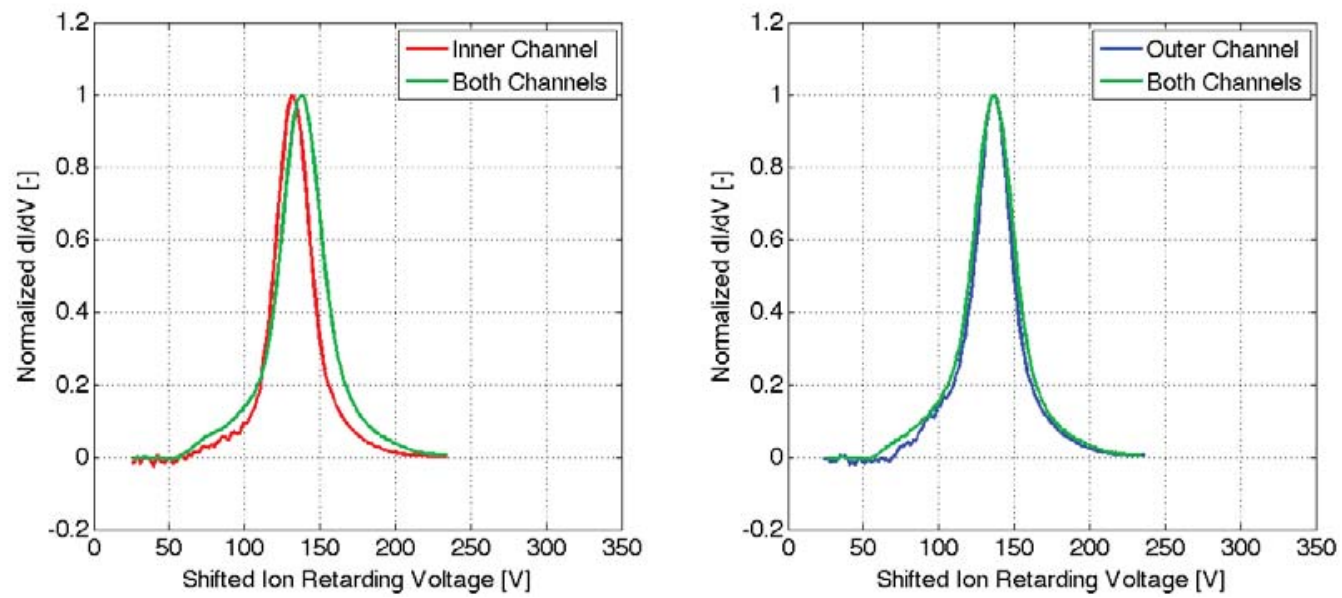

(c) Corrected and differentiated RPA traces at $100 \%$ nominal flow rate

Figure 15: Single-channel and dual-channel differentiated RPA traces at fixed discharge voltage (150 V). Ion retarding voltages were shifted by the corresponding plasma potentials to place the peaks at the accelerating voltages. Dual-channel spectra overlaid with inner-channel spectra were taken with the RPA and LP aligned with the inner-channel centerline, while dual-channel spectra overlaid with outer-channel spectra were taken in alignment with the outer-channel centerline. 


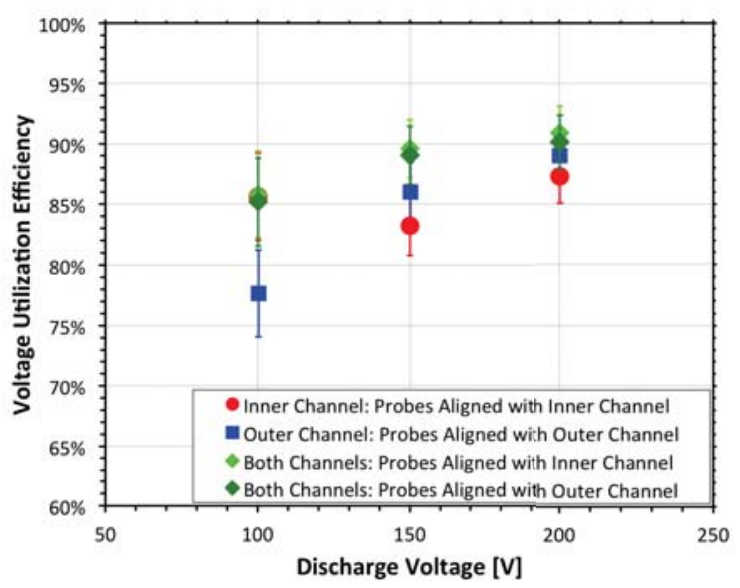

(a) Voltage utilization efficiency variation with voltage

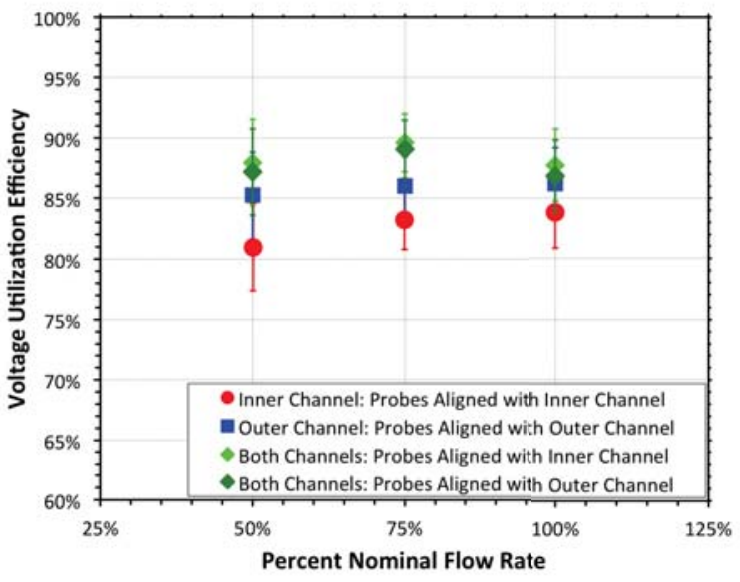

(b) Voltage utilization efficiency variation with mass flow rate

Figure 16: Voltage utilization efficiencies

\section{Discussion}

The results of the far-field diagnostics show that the X2 NHT behaves in a similar manner as a typical Hall thruster in the low-voltage, high thrust-to-power operating regime. The far-field Faraday probe measurements yield typical low-voltage current density profiles, and the $\mathrm{E} \times \mathrm{B}$ and RPA measurements by themselves do not yield anything unique when both channels are operating at the same discharge voltage. The values for all utilization efficiencies are within expected ranges, and the trends with respect to discharge voltage and flow rate are typical as well.

The comparison between measured total efficiencies and calculated total efficiencies based on the existing models are shown in Figures 17(a) and 17(b). The absolute difference between the measured and calculated total efficiencies vary from $1 \%$ to $6 \%$. Given the small difference, the results of the far-field measurements also show that existing efficiency models can be applied to an NHT with only minor modifications to Faraday probe analysis. The uncertainties of the calculated $\eta_{t}$ are large due to the compounding uncertainties from all of the contributing efficiencies.

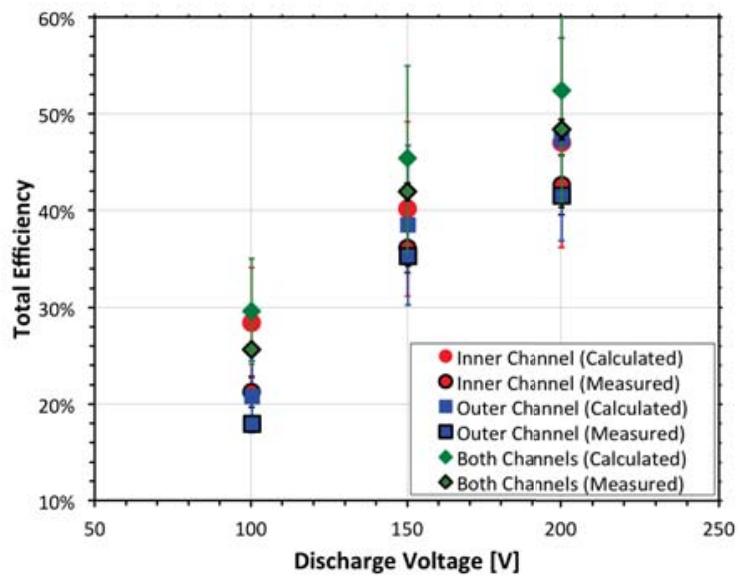

(a) Variation of total efficiencies with discharge voltage

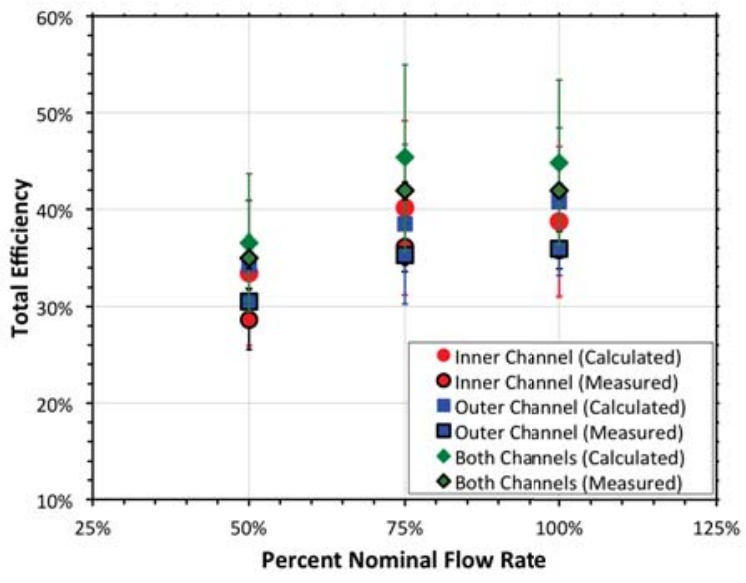

(b) Variation of total efficiencies with mass flow rate

Figure 17: Total efficiencies

The small gains in performance from the X2's dual-channel operation, as illustrated in Figure 2, are most likely due to the increased voltage utilization, and this phenomenon appears in $\mathrm{E} \times \mathrm{B}$ measurements as well. The higher acceleration voltages in dual-channel mode resulted in shifts in $\mathrm{E} \times \mathrm{B}$ measurements, as seen previously in all $\mathrm{E} \times \mathrm{B}$ spectra. Shifts to higher applied voltages correspond to shifts to higher ion velocities, as the applied voltage is directly 
proportional to the velocity of the ion passing through the $\mathrm{E} \times \mathrm{B}$ probe's test section.

Examination of cathode coupling voltages, $V_{c}$, also suggest improved voltage utilization in dual-channel mode. Cathode coupling voltage is the voltage used to extract electrons from the cathode, and although this process is necessary, the extraction of electrons does not directly contribution to ion acceleration. In order to maximize $\eta_{v}, V_{c}$ is preferably minimized so that $V_{a}$ can be a larger fraction of $V_{d}$. $V_{c}$ is calculated from the difference of the downstream plasma potential and the cathode floating voltage. Both measurements were taken with respect to facility ground. The values of $V_{p}$ were the same as those used to correct the RPA measurements and were measured by the far-field Langmuir probe.

Calculated cathode coupling voltages are shown in Figures 18(a) and 18(b). Although $V_{c}$ appears to increase with discharge voltage, the fraction $V_{c} / V_{d}$ actually decreases with discharge voltage. This trend agrees with the increase in voltage utilization with increasing discharge voltage. In comparison to $V_{c}$ in single-channel mode, the cathode coupling voltages are consistently lower in the dual-channel mode. As a result, more of the discharge voltage is available to accelerate ions in dual-channel mode. Additional experiments will be necessary to further investigate the impact of cathode coupling on the dual-channel mode.

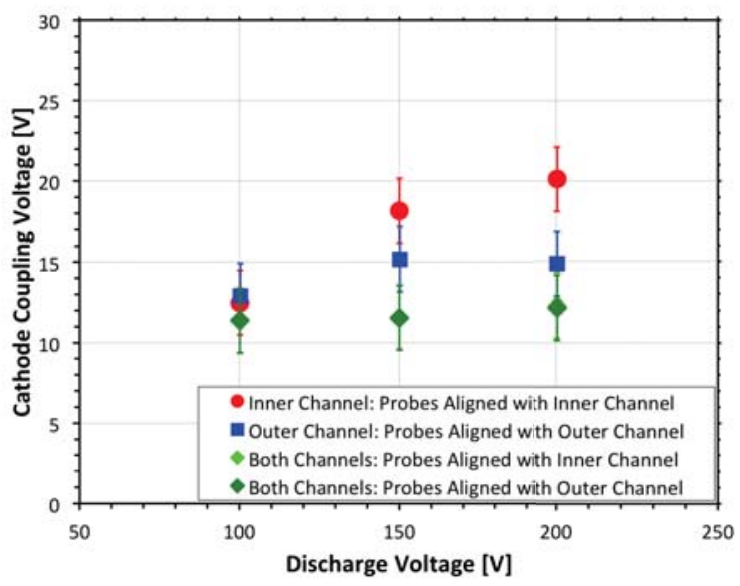

(a) Variation of cathode coupling voltage with discharge voltage

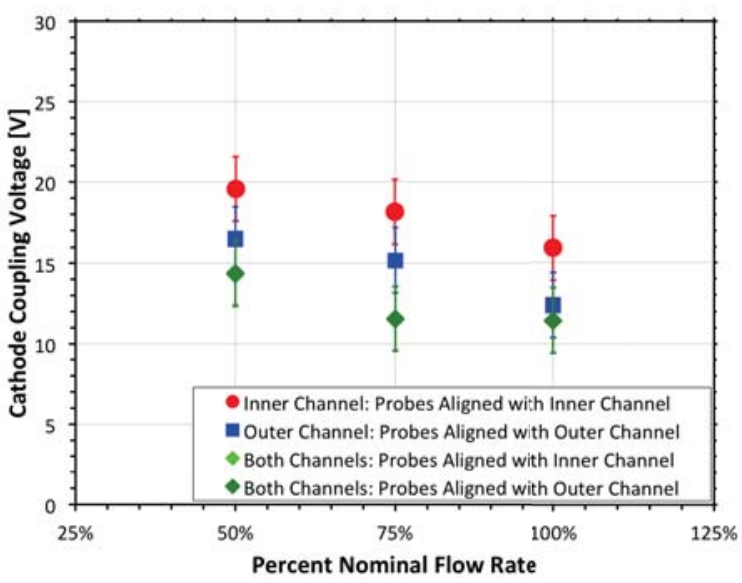

(b) Variation of cathode coupling voltage with mass flow rate

Figure 18: Cathode coupling voltages

The increase in voltage utilization is one of the unique features of the dual-channel mode, and its occurrence shows that the two channels can interact in an advantageous manner. However, at present, the gains are relatively small but may be exploitable with further study.

\section{Conclusion}

Far-field plume measurements were performed on the X2 nested-channel Hall thruster using an array of diagnostics in order to study how the individual channels combine during simultaneous operation. The diagnostics utilized during this study include a nude Faraday probe, retarding potential analyzer, and E $\times$ B probe. Data from these probes were used to calculate the following utilization efficiencies that contribute to the total efficiency of the thruster:

- Current utilization efficiency, $\eta_{b}$

- Divergence efficiency, $\eta_{d}$

- Charge utilization efficiency, $\eta_{q}$

- Voltage utilization efficiency, $\eta_{v}$

- Total mass utilization efficiency, $\eta_{M}$

Comparisons of utilization efficiencies during dual-channel mode against those of single-channel mode show an increase in voltage utilization. The increase in voltage utilization consistently corresponded to a decrease in cathodecoupling voltage. Current utilization, divergence, charge utilization, and mass utilization efficiencies during dualchannel operation remained near the values measured during single-channel operation. 
A nested-channel Hall effect thruster has been shown to operate much like a conventional Hall thruster when all available channels are at the same discharge voltage. One unique feature of the NHT is the improved ion acceleration with both channels active. Subsequent investigation will focus on the internal and very-near-field plasma potential distribution of the X2 in order to gain further understanding of this phenomenon.

\section{Acknowledgements}

The authors would like to thank our sponsors at the Air Force Research Laboratory, including Dr. Daniel Brown, Dr. Brian Beal, and Dr. James Haas. Additional insight regarding Faraday probes, low-voltage Hall thruster operation, and efficiency analysis from Dr. Brown was greatly appreciated. We would also like to thank the AFRL for the new $\mathrm{LaB}_{6}$ cathode and RPA used in our experiments. Thanks go to Rohit Shastry, Roland Florenz, Adam Shabshelowitz, Käthe Dannenmayer du Centre National de la Recherche Scientifique, and Cameron Lee of Cornell University for their assistance with the experiments performed for this study. Raymond Liang is supported by the National Defense Science and Engineering Graduate (NDSEG) Fellowship.

\section{Appendix: Mixed-Voltage Operation}

The majority of the operating conditions used during testing of the $\mathrm{X} 2$ have matching discharge voltages and current densities between the two channels. However, operation with mixed discharge voltages has also been investigated with a small number of additional conditions. No mixed-voltage condition has yielded a particularly unique performance advantage, and, in most cases, these operating modes have comparable performance to matched-voltage conditions. However, a far-field RPA and $\mathrm{E} \times \mathrm{B}$ probe have produced some unique measurements for mixed-voltage conditions.

This appendix shows the results of RPA and $\mathrm{E} \times \mathrm{B}$ measurements for the flow rate combination of $7.0 \mathrm{mg} / \mathrm{s}$ through the inner channel and $17.4 \mathrm{mg} / \mathrm{s}$ through the outer channel. The discharge voltage applied to the inner channel was fixed at $150 \mathrm{~V}$, and the outer channel discharge voltage was increased to $200 \mathrm{~V}$ and $300 \mathrm{~V}$. The cathode flow fraction was fixed at $10 \%$, and the electromagnets were tuned to maximize efficiency at each operating condition.

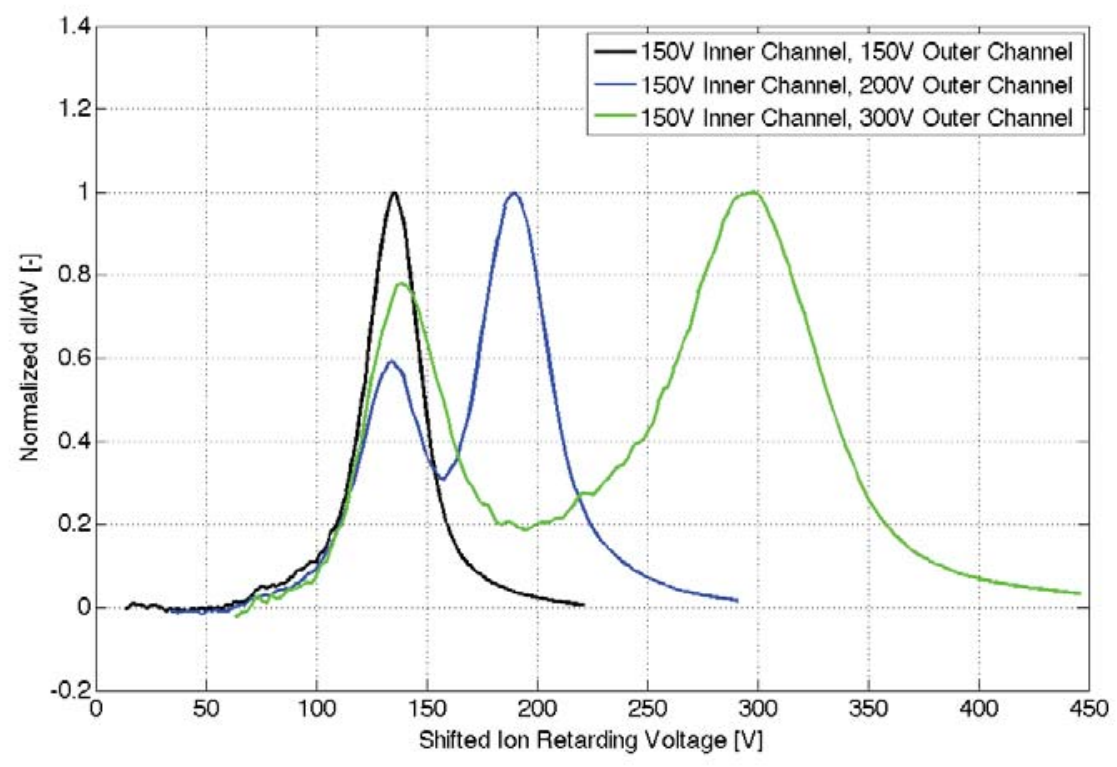

Figure 19: RPA measurements for mixed-voltage operating conditions

Figure 19 shows processed RPA measurements which include smoothing of the differentiated signal and voltage correction for local plasma potential. The sweeps shown in Figure 19 were taken with the RPA aligned with the outer channel centerline. Measurements during the mixed-voltage conditions show bimodal distributions with peaks near the two applied discharge voltages. At a distance of $2.1 \mathrm{~m}$, the RPA clearly collects ions from both channels. Current collected from the inner channel has a weaker signal due to the lower total current of the inner channel and the probe's 
alignment. However, both peaks remain well-defined and the two acceleration voltages can be measured from RPA traces taken during a single mixed-voltage condition.

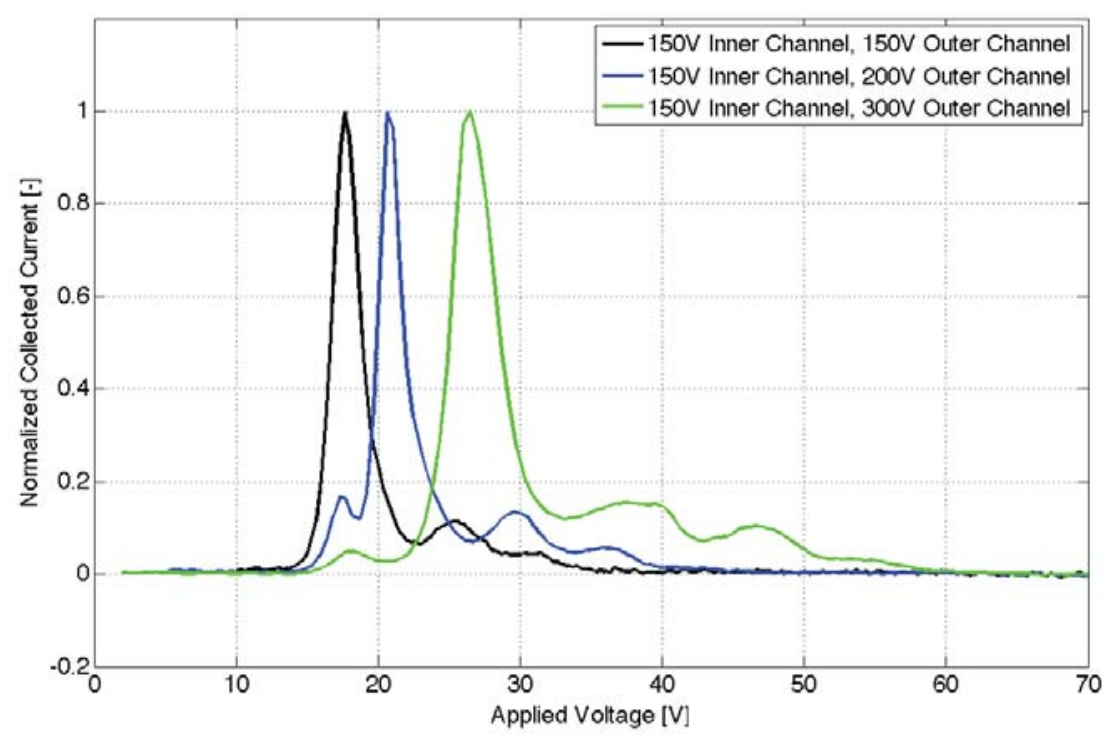

Figure 20: $\mathbf{E} \times \mathbf{B}$ measurements for mixed-voltage operating conditions
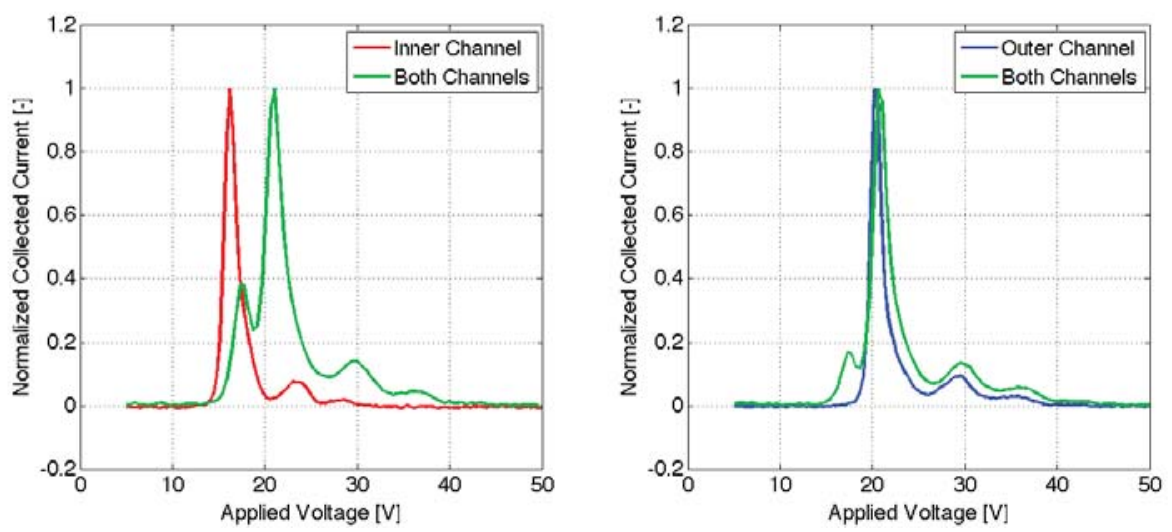

Figure 21: Single-channel and dual-channel $\mathbf{E} \times \mathbf{B}$ measurements for a mixed-voltage operating condition (150V inner channel, $200 \mathrm{~V}$ outer channel, $75 \%$ nominal flow rate)

Figure 20 shows the evolution of the $\mathrm{E} \times \mathrm{B}$ spectra as the outer discharge voltage is increased to $300 \mathrm{~V}$. The dominant peaks in the spectra shift to higher voltages, as expected. In the mixed-voltage spectra, smaller peaks are present at voltages lower than the dominant peaks. As shown in Figure 21, this smaller peak corresponds to the singly-charged ion population from the inner channel. Having fallen through a smaller potential drop, the inner channel ions register as a small population of slower ions in the dual-channel spectra.

Figure 21 also shows that multiply-charged species from the inner channel are not resolved in the dual-channel spectra, and therefore, quantifying species fractions becomes slightly more involved during mixed-voltage operation. The inner-channel signal is obscured by the higher signal provided by the outer channel, and in order to approximate the multiply-charged species fractions of the inner channel, single-channel measurements had to be taken. Error in current densities can be significant, as the creation of multiply-charged species increases in dual-channel mode. However, it is possible that, if the discharge voltage difference were sufficiently large between the two channels, all multiply-charged peaks from both channels would appear on an E×B spectrum. This would require the singly-charged ions from the outer channel to move faster than the triply-charged or quadruply-charged ions from the inner channel. 


\section{References}

${ }^{1}$ Jacobson, D.T., John, J.W., Kamhawi, H., Manzella, D.H, and Peterson, P.Y., “An Overview of Hall Thruster Development at NASA's John H. Glenn Research Center," AIAA/ASME/SAE/ASEE Joint Propulsion Conference and Exhibit, Tucson, AZ, July 2005, pp. 1-15.

${ }^{2}$ Spores, R., Monheiser, J., Dempsey, B.P., Wade, D., Creel, K., Jacobson, D., and Drummond, G., "A Solar Electric Propulsion Caro Vehicle to Support NASA Lunar Exploration Program," International Electric Propulsion Conference, Princeton, NJ, October 2005, pp. 1-22.

${ }^{3}$ Wilson, F., "Propulsion and Energy: Electric Propulsion," Aerospace America, December 2006.

${ }^{4}$ Brown, D.L., Beal, B.E., and Haas, J.M., "Air Force Research Laboratory High Power Electric Propulsion Technology Development," IEEE Aerospace Conference, Big Sky, MT, March 2010, pp. 1-9.

${ }^{5}$ Hofer, R.R., Katz, I., Mikellides, I.G., Goebel, D.M., Jameson, K.K., Sullivan, R.M., and Johnson, L.K, "Efficacy of Electron Mobility Models in Hybrid-PIC Hall Thruster Simulations," AIAA/ASME/SAE/ASEE Joint Propulsion Conference and Exhibit, Hartford, CT, July 2008, pp. $1-29$.

${ }^{6}$ Brown, D.L., Larson, C.W., Beal, B.E., and Gallimore, A.D., "Methodology and Historical Perspective of a Hall Thruster Efficiency Analysis," Journal of Propulsion and Power, Vol. 25, No. 6, 2009, pp. 1163-1177.

${ }^{7}$ Brown, D.L. and Gallimore, A.D., "Evaluation of Ion Collection Area in Faraday Probes," Review of Scientific Instruments, Vol. 81, No. 063504, 2010, pp. 1-11.

${ }^{8}$ Brown, D.L. and Gallimore, A.D., "Evaluation of Facility Effects on Ion Migration in a Hall Thruster Plume," Journal of Propulsion and Power, 2011, Accepted for publication.

${ }^{9}$ Shastry, R., Hofer, R.R., Reid, B.M, and Gallimore, A.D., "Method for Analyzing E×B Probe Spectra from Hall Thruster Plumes," Review of Scientific Instruments, Vol. 80, No. 063502, 2009, pp. 1-11.

${ }^{10}$ Hofer, R.R., Development and Characterization of High-Efficiency, High-Specific Impulse Xenon Hall Thrusters, Ph.D. thesis, University of Michigan, Ann Arbor, MI, 2004.

${ }^{11}$ Beal, B.E., Clustering of Hall Effect Thrusters for High-Power Electric Propulsion Applications, Ph.D. thesis, University of Michigan, Ann Arbor, MI, 2005.

${ }^{12}$ Walker, M.L.R. and Gallimore, A.D., "Performance Characteristics of a Cluster of 5-kW Laboratory Hall Thrusters," Journal of Propulsion and Power, Vol. 23, No. 1, 2007, pp. 35-43.

${ }^{13}$ Reid, B.M., Shastry, R., Gallimore, A.D., and Hofer, R.R., "Angulary-Resolved E×B Probe Spectra in the Plume of a 6-kW Hall Thruster," AIAA/ASME/SAE/ASEE Joint Propulsion Conference and Exhibit, Hartford, CT, July 2008, pp. 1-21.

${ }^{14}$ Reid, B.M., The Influence of Neutral Flow Rate in the Operation of Hall Thrusters, Ph.D. thesis, University of Michigan, Ann Arbor, MI, 2009.

${ }^{15}$ Varian, "Vacuum Measurement Catalog," 2010

${ }^{16}$ Brown, D.L., Investigation of Low Discharge Voltage Hall Thruster Characteristics and Evaluation of Loss Mechanisms, Ph.D. thesis, University of Michigan, Ann Arbor, MI, 2009. 